\title{
Pulse-Power-Detection Analysis of Incoherent O-CDMA Systems Under the Influence of Fiber Temperature Fluctuations
}

\author{
Chun-Yi Tsai, Guu-Chang Yang, Fellow, IEEE, Jing-Shiuan Lin, Cheng-Yuan Chang, Member, IEEE, \\ Ivan Glesk, Senior Member, IEEE, and Wing C. Kwong, Senior Member, IEEE
}

\begin{abstract}
In this paper, a pulse-shift technique, which divides every time slot (or chip) into equal-width sub-chips, is used to model the effect of fiber temperature fluctuations in incoherent (asynchronous) optical code-division multiple-access (O-CDMA) systems. With the advances in all-optical thresholding technology, power detection of ultrashort optical pulses is possible. This paper also formulates a new pulse-power-detection model for incoherent O-CDMA and applies it to the analysis of the pulseshift technique. Numerical studies and computer simulations are presented to validate the new analytical model. Our study shows that the pulse-power model results in better performance than the conventional pulse-energy model in incoherent O-CDMA.
\end{abstract}

Index Terms-optical code division multiple access, optical fiber communications, temperature fluctuation, time skew

\section{INTRODUCTION}

$\mathbf{O}$ PTICAL code-division multiple access (O-CDMA) has recently attracted interests in high-speed fiber-optic systems and networks because it carries desirable characteristics, such as dynamic bandwidth assignment, efficient in bursty traffic, soft limit in the number of subscribers, and gradual performance degradation as a function of the number of simultaneous users [1]-[9]. With the advances in technology, multiuser experimental testbeds operating at $10 \mathrm{Gbit} / \mathrm{s}$ have been demonstrated [10]-[12]. The choice of suitable optical codes is important in the design of incoherent (or directdetection) O-CDMA systems because the codes affect spectral efficiency, capacity, and the amount of multiple-access interference (MAI), which, in turn, determines system performance.

Manuscript received October 8, 2016; revised February 16, 2017; accepted March 31, 2017. The associate editor coordinating the review of this paper and approving it for publication was ?. ?????. This work was supported in part by the Ministry of Science and Technology of Republic of China under Grants MOST 105-2221-E-005-007-MY3 and MOST 103-????-E-???-???, in part by the U.S. Defense Advance Research Projects Agency under Grant MDA972-03-1-0006, and in part by the Faulty Development and Research Grants of Hofstra University.

C.-Y. Tsai, G.-C. Yang, and J.-S. Lin are with the Department of Electrical Engineering and the Graduate Institute of Communication Engineering, National Chung Hsing University, Taichung 402, Taiwan. (email: newfju2014@gmail.com; gcyang@dragon.nchu.edu.tw; bill79910@gmail.com).

C.-Y. Chang is with the Department of Electrical Engineering, National United University, Miaoli, Taiwan. (email: chengyuan@nuu.edu.tw).

I. Glesk is with the Center for Intelligent and Dynamic Communications, Electronic and Electrical Engineering Department, University of Strathclyde, Glasgow, G1 1XW, United Kingdom. (email: ivan.glesk@eee.strath.ac.uk).

W.C. Kwong is with the Department of Engineering, Hofstra University, Hempstead, NY 11549. (email: eggwck@hofstra.edu).

Digital Object Identifier 10.1109/JLT.2017.??????
To minimize the impact of MAI, two-dimensional (2-D) wavelength-time codes with low cross-correlation functions (of at most one) have been designed. In addition to code length and weight, this kind of optical codes allows the number of wavelengths to be adjustable for performance improvement [1]-[3], [13]-[15]. One good example is the family of carrier-hopping prime codes (CHPCs) because they have cross-correlation functions of at most one (for minimal MAI) and zero autocorrelation sidelobes (for ease of code tracking) [1]-[3]. Moreover, their algebraic construction algorithm can easily be modified to generate various families of 2-D codes with expanded cardinality or different code properties [3], [6].

Recently, the effect of environmental temperature fluctuations to incoherent ${ }^{1}$ fiber-optic O-CDMA systems was studied [16]. While fiber temperature fluctuations can cause broadening and time skew in optical pulses, their influence is pronounced in 2-D wavelength-time codes because multiwavelength pulses in codewords see different amounts of time skew. Osadola, et al. [16] showed that these two phenomena distorted the autocorrelation function and worsened the system performance. Moreover, Lin, et al. [9] found that the crosscorrelation function could also be affected, and this should also be considered whenever there existed time skews in the multiwavelength pulses of codewords. To accurately account for the effect of fiber temperature fluctuations to 2-D wavelengthtime codes, a new analytical model of performance will be needed. To achieve this goal, the pulse-shift technique in [9] is modified in this paper by borrowing the concept of chip granularity. The concept subdivides every time slot (or chip) in each codeword into sub-chips of equal width. The time skews in the multiwavelength pulses of codewords can then be quantified as sub-chip shifts. For the first time, the effect of fiber temperature fluctuations is modeled as a function of chip granularity in this paper.

\footnotetext{
${ }^{1}$ Traditionally, the term "incoherent" refers to an O-CDMA system that does not require codewords from simultaneous users to be synchronized in the bit (or frame) level. Also known as "asynchronous" O-CDMA, this multiaccess technique doesn't require users to coordinate their transmissions, and they can transmit their codewords at any time. For ease of mathematics, most of the papers in incoherent (asynchronous) O-CDMA assumed that the codewords were synchronized in the "chip" level [1]-[5]. In the study of the effect of fiber temperature fluctuations, this paper shows that the amounts of time skew added to the pulses of codewords can be modeled in the terms of "sub-chip." Thus, the proposed pulse-shift technique and the analytical models in this paper assume that the synchronization requirement is further relaxed to the "sub-chip" level.
} 
Due to the response-time and bandwidth limitations of optoelectronic devices (e.g., photodetectors), the "power" of optical pulses in the picosecond scale could only be detected as "energy" over several chips [2]. So, the conventional detection/thresholding process in incoherent O-CDMA receivers relied on the discrimination of the energy of a narrow autocorrelation peak against the energy of the accumulated crosscorrelation function (from interfering codewords) spreading over several chips. Even though the cross-correlation function is usually low in power, the energy (over several chips) can occasionally be as large as that of the autocorrelation peak. As a result, the pulse-energy detection will have a tendency of wrongly recovering data bits of 0 to 1 . On the other hand, by discriminating the actual power (i.e., height) of the autocorrelation peak from that of the cross-correlation function within one chip, pulse-power detection can better measure the actual impact of MAI and results in more realistic performance measurement.

With the invention of all-optical thresholders [17]-[20], pulse-power detection is possible in incoherent (asynchronous) O-CDMA. Two kinds of all-optical thresholders and their nonlinear characteristics on the performances of ultrashort opticalpulse communication and spectrally-phase-encoded (SPE) OCDMA systems were analytically and statistically studied [21]-[23]. In these studies, the physical models of these alloptical thresholders were considered, along with the thermal, shot, beat, and amplified-spontaneous-emission noises. Statistical models of the probability density functions that tied to the actual physical devices in use were applied. Moreover, Zefreh and Salehi [23] included the effect of MAI in a SPEOCDMA system in the statistical model. Perfect spectralphase-code decoding and code independency were assumed in order to study the actual effect of all-optical thresholders to the system performance. This paper has several aspects that make it different from [21]-[23]. No perfect decoding, code independency, the specific kind of all-optical thresholders in use, or physical-layer modeling is assumed. Functioning in a different principle from SPE-OCDMA, incoherent O-CDMA is best to operate in a MAI-limited environment [24]. Thus, this paper focus on the effect of fiber temperature fluctuations to the code properties. The statistical models in [21]-[23] will be perfect add-ons to this work. Together they can provide a complete physical-layer model of an incoherent $\mathrm{O}$ CDMA system under the combined effects of fiber temperature fluctuations and all-optical thresholders.

There are two themes in this paper: study the fibertemperature-fluctuation effect and the pulse-shift technique under pulse-power and pulse-energy detection in incoherent (asynchronous) O-CDMA systems. Section II reviews an incoherent (asynchronous) O-CDMA system utilizing the pulsepower detection with all-optical thresholders. Moreover, the effect of fiber temperature fluctuations to the auto- and crosscorrelation functions of 2-D wavelength-time codes, such as the CHPCs, are investigated. Besides deteriorating the autocorrelation peak, the time skews in the multiwavelength pulses of codewords also affect the cross-correlation functions. Section II further shows that the time skews can be modeled as chip granularity [9]. In Section III, new pulse-shift analytical

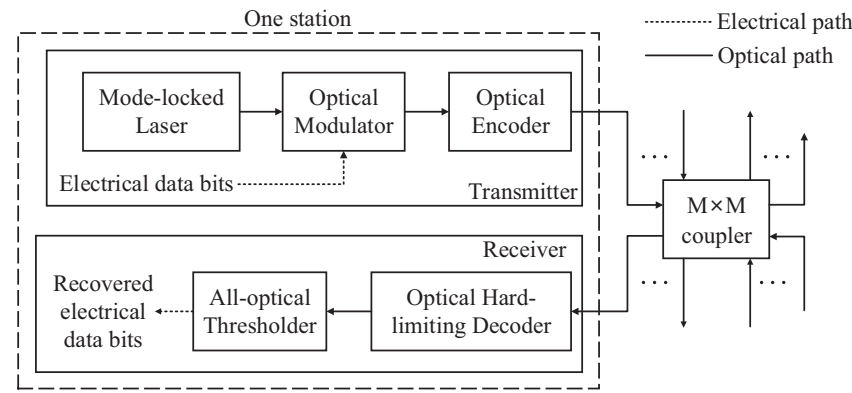

Fig. 1. Block diagram of a typical incoherent (asynchronous) O-CDMA system.

models under pulse-power and pulse-energy detection are formulated. In Section IV, numerical examples and computer simulations are presented to validate the models. Our study shows that the power-detection model gives more realistic and better performance than the conventional energy-detection model. Finally, a conclusion is given in Section V.

\section{System Model AND BACKGROUND}

In incoherent O-CDMA systems with on-off keying (OOK) modulation, a user conveys the address codeword of its intended receiver whenever a data bit of 1 is transmitted, but nothing is conveyed for a data bit of 0 . The notation $\left(L \times N, w, \lambda_{a}, \lambda_{c}\right)$ is here used to represent a family of 2D wavelength-time codes of $L$ wavelengths, $N$ time slots (i.e., code length), $w$ pulses (i.e., code weight), the maximum autocorrelation sidelobe $\lambda_{a}$, and the maximum periodic cross-correlation value $\lambda_{c}$. The $w$ multiwavelength pulses of each 2-D codeword can be represented as $w$ ordered pairs $\left[\left(\lambda_{0}, t_{0}\right),\left(\lambda_{1}, t_{1}\right), \ldots,\left(\lambda_{j}, t_{j}\right), \ldots,\left(\lambda_{w-1}, t_{w-1}\right)\right]$, where each ordered pair denotes that the pulse of wavelength $\lambda_{j}$ is located at time-slot (or chip) location $t_{j} \in[0, N-1]$.

\section{A. Incoherent (Asynchronous) O-CDMA System Model}

Fig. 1 shows an incoherent (asynchronous) O-CDMA system model. The system consists of $M$ stations (or users) linking to a $M \times M$ coupler via optical fibers [1]-[3]. The coupler is a shared medium used to collect/distribute OCDMA waveforms from/to all stations. Each station contains a pair of optical transmitter and receiver. In the transmitter, a mode-locked laser is used to generate a train of narrow optical pulses with a repetition rate of one pulse per bit period [2], [12]. Whenever there is a "1" in the electrical data-bit stream, the optical modulator will be opened to pass one such pulse to the optical encoder. This gated pulse is then split and time delayed in the optical encoder to generate the optical (address) codeword of the intended station.

The role of the receiver is to decode the arrival codewords, distinguish the correct codeword from the interfering codewords, and finally recover the transmitted data bits. The optical decoder serves as an inverted filter of its associated optical encoder, and thus they are constructed using similar hardware. If an arrival codeword matches with the address codeword of the optical decoder, an autocorrelation function with a high 

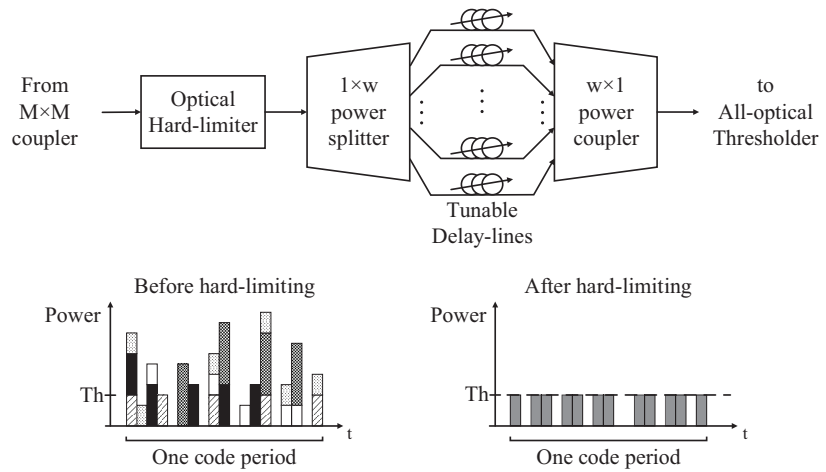

(a)

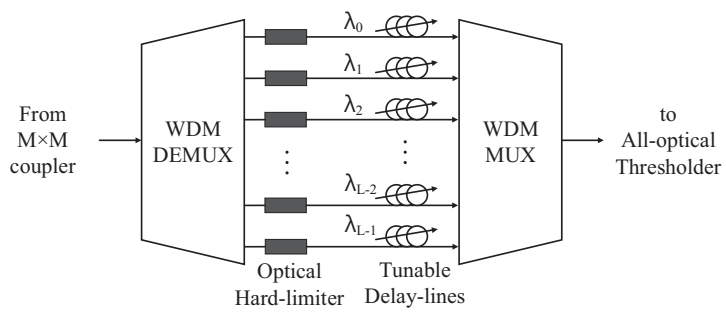

(b)

Fig. 2. Optical hard-limiting decoder: (a) 1-D design; (b) 2-D design [30].

peak of height $w$ will be created. This is because the $w$ pulses of the correct codeword are realigned on top of each other, all within one chip. On the other hand, an incorrect (i.e., interfering) codeword will give a low cross-correlation function at the decoder. For example, the CHPCs are designed to have $\lambda_{c}=1$ in order to minimize the MAI [1]-[3].

It is known that optical hard-limiters [25], [26] can be placed in an incoherent O-CDMA receiver to reduce the effects of MAI and near-far problem and, in turn, to improve system performance [1], [3], [27]-[30]. Fig. 2 shows the designs of 1-D and 2-D optical hard-limiting decoders. For example, making use of the short carrier-recovery time and high gain of semiconductor optical amplifiers (SOAs), Kanellos, et al. [25] demonstrated an optical hard-limiter using a deeply saturated SOA-based interferometric gate and studied the power transfer function of the design. Power equalization was achieved by clamping the output power to a constant level for different input power levels that are greater than a specific (power) threshold level $T h$. Due to the SOA gain dynamics, the 2-D design in Fig. 2(b) requires the optical hard-limiters be placed along the individual wavelength paths [30].

Because narrow optical pulses (in tens or few picoseconds) are used in high-speed incoherent (asynchronous) O-CDMA systems [2], [10]-[12], the detection of the autocorrelation peak (within one chip) cannot simply be done by optoelectronic devices (e.g., photodetectors) because they tend to measure the energy (over several chips), instead of the instantaneous power [20], [31]. Although the accumulated cross-correlation function from multiple interfering codewords usually carries lower power (per chip) than the autocorrelation peak, the energy (over several chips) can occasionally be as strong as that of the autocorrelation peak. As a result, a threshold detector relying on the pulse-energy detection has a higher tendency of wrongly recovering data bits of 0 to 1 . The all-optical thresholders overcome this problem and make the power detection of narrow autocorrelation peaks in the optical domain possible. They have been applied in ultrashort-pulse O-CDMA testbeds [17]-[20]. A new pulse-power-detection analytical model, which discriminate the actual power (i.e., height) of the autocorrelation peak from that of the accumulated cross-correlation function, is formulated in Section III.

\section{B. Effect of Fiber Temperature Fluctuations}

Due to environmental temperature fluctuations, optical pulses traveling in long length of optical fiber (e.g., an underground or transoceanic link) will get broadened and time-skewed, even though the fiber-optic link is fully compensated for power loss and chromatic dispersion. Osadola, et al. [16] studied the effect of fiber temperature fluctuations in high-speed incoherent O-CDMA systems with picosecond multiwavelength pulses. As the fiber temperature may vary by $20^{\circ} \mathrm{C}$ or higher, the fiber thermal coefficient, which has a typical value of $D_{\text {temp }}=0.0025 \mathrm{ps} / \mathrm{nm} / \mathrm{km} /{ }^{\circ} \mathrm{C}$ for a nonzero dispersion-shifted fiber, can severely distort the characteristics (e.g., height, shape, width, and chip locations) of the correlation functions at the optical decoder. They, in turn, affect the threshold-decision process and deteriorate system performance.

Taking into consideration of fiber thermal coefficient $D_{\text {temp }}$ (ps $\left./ \mathrm{nm} / \mathrm{km} /{ }^{\circ} \mathrm{C}\right)$, the temperature-induced time skew $\Delta t(\mathrm{ps})$ and broadening $\Delta \sigma$ (ps) of multiwavelength pulses can generally be expressed as [16]

$$
\begin{aligned}
& \Delta t=D_{\text {temp }} \times \Delta T \times \Delta \Lambda \times d \quad(\mathrm{ps}) \\
& \Delta \sigma=D_{\text {temp }} \times \Delta T \times \Delta \lambda \times d(\mathrm{ps})
\end{aligned}
$$

where $\Delta T\left({ }^{\circ} \mathrm{C}\right)$ is the average change in temperature experienced in the fiber of length $d(\mathrm{~km}), \Delta \Lambda(\mathrm{nm})$ is the spectral spacing between two adjacent wavelengths, and $\Delta \lambda(\mathrm{nm})$ is the spectral linewidth of the multiwavelength pulses.

Assume that the multiwavelength pulses from the modelocked laser are modeled with a hyperbolic-secant-squared $\left(\mathrm{sech}^{2}\right)$ pulse envelope. From (1) and (2), the (normalized) $\operatorname{sech}^{2}$ pulse envelope after an optical pulse (of one wavelength) experienced $\Delta T$ average temperature change in a fiber of length $d$ can be expressed as

$$
S(t)=\frac{\sigma}{\sigma+\Delta \sigma} \operatorname{sech}^{2}\left(\frac{t-k \Delta t}{\sigma+\Delta \sigma}\right)
$$

where $k \in[0, L-1]$ denotes the index of the $k$ th wavelength $\lambda_{k}$. Without temperature changes (i.e., $\Delta \sigma=\Delta t=0$ ), the pulse in (3) is normalized to have a peak power of 1 and the full-width at half-maximum (FWHM) duration is about 1.76 times the parameter $\sigma$, which is called the duration of the $\operatorname{sech}^{2}$ pulse.

Without any loss of generality, this paper assumes that $\lambda_{0}<$ $\lambda_{1}<\cdots<\lambda_{L-1}$ and they are equally spectral-spaced by $\Delta \Lambda$. The time skew of the pulse of wavelength $\lambda_{k}$ increases as a function of $k$ due to the term $k \Delta t$ in (3). In other words, the time skew of a pulse of longer wavelength will be larger and 

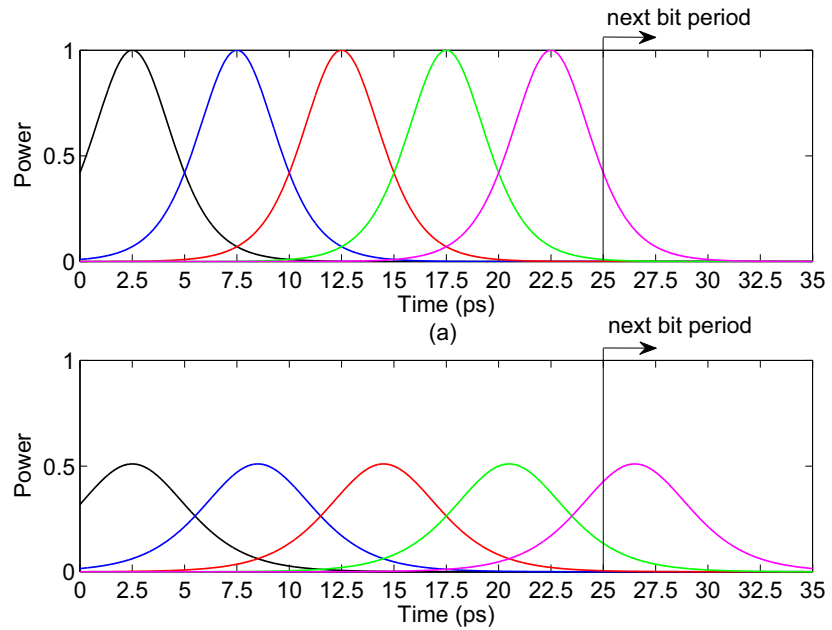

(b)

Fig. 3. $(5 \times 5,5,0,1)$ CHPC codeword $X_{1}=[(0,0),(1,1),(2,2),(3,3)$, $(4,4)]$ of chip-width $t_{c}=5$ ps after traveling in a fiber of $d=20 \mathrm{~km}$ and experiencing (a) $\Delta T=0^{\circ} \mathrm{C}$ and (b) $\Delta T=20^{\circ} \mathrm{C}$ of temperature changes. (One color represents one wavelength.)

proportional to $k$. Also, a pulse of any wavelength is broadened in time due to the term $\sigma+\Delta \sigma$ in (3). However, the amounts of broadening are found the same for all pulses because they are assumed to have identical spectral linewidth $\Delta \lambda$. To maintain the same total area under curve (i.e., energy), the peak power of a pulse decreases as the pulse gets broadened, as indicated in the term $\sigma /(\sigma+\Delta \sigma)$ in (3).

In the following illustration, the $(5 \times 5,5,0,1)$ CHPCs are used. They consist of $N=5$ time slots (or chips), $L=5$ wavelengths, and $w=5$ mark chips. They are also assumed to have chip-width $t_{c}=5 \mathrm{ps}$, pulse parameter $\sigma=2.5$ (i.e., FWHM width $=1.76 \times 2.5=4.4 \mathrm{ps}$ ), spectral linewidth $\Delta \lambda=1 \mathrm{~nm}$, spectral spacing $\Delta \Lambda=1 \mathrm{~nm}$, and fiber thermal coefficient $D_{\text {temp }}=0.0025 \mathrm{ps} / \mathrm{nm} / \mathrm{km} /{ }^{\circ} \mathrm{C}$. Fig. 3 shows the codeword $X_{1}=[(0,0),(1,1),(2,2),(3,3),(4,4)]$ after traveling in a fiber of $d=20 \mathrm{~km}$ and experiencing (a) $\Delta T=0^{\circ} \mathrm{C}$ and (b) $\Delta T=20^{\circ} \mathrm{C}$ of temperature changes. So, Fig. 3(a) resembles to the original codeword without the influence of fiber temperature fluctuations (i.e., $\Delta t=0$ and $\Delta \sigma=0$ ). Fig. 3(b) shows that the broadened and timeskewed pulses of the distorted codeword with $\Delta t=1 \mathrm{ps}$ and $\Delta \sigma=1 \mathrm{ps}$, calculated from (1) and (2). The pulses are broadened to $1.76(\sigma+\Delta \sigma)=6.2$ ps (FWHM) with the normalized peak power reduced to 0.51. Also from (3), the amount of time skew depends on the pulse wavelength.

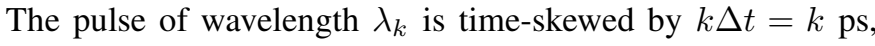
where $k \in[0,4]$. So, the amounts of time skew added to the pulses of wavelengths $\lambda_{0}, \lambda_{1}, \lambda_{2}, \lambda_{3}$, and $\lambda_{4}$ in the distorted codeword of $X_{1}$ are $0,1,2,3$, and $4 \mathrm{ps}$, respectively, as shown in Fig. 3(b).

If a codeword arrives at its intended decoder, all its $w$ pulses will be realigned on top of each other and an autocorrelation peak of $w$ can be obtained in one chip. Using the $\operatorname{sech}^{2}$ pulse model in (3), the envelope of the autocorrelation peak under the influence of fiber temperature fluctuations can be computed

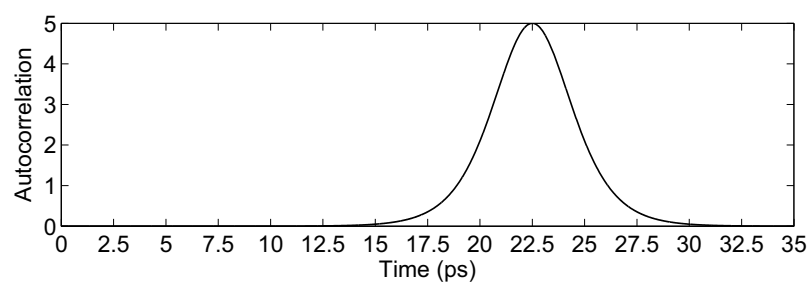

(a)

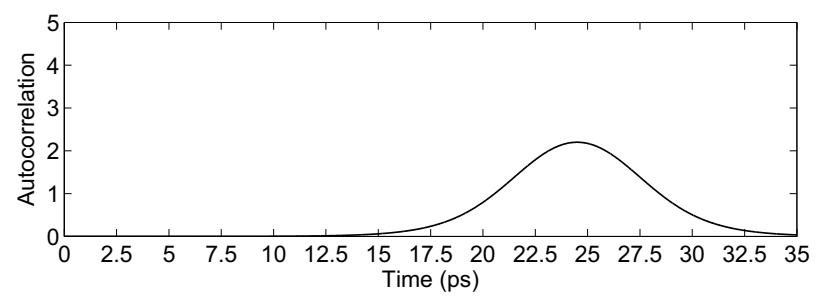

(b)

Fig. 4. Autocorrelation function of $X_{1}$ of chip-width $t_{c}=5 \mathrm{ps}$ after $X_{1}$ has traveled in a fiber of $d=20 \mathrm{~km}$ and experienced (a) $\Delta T=0^{\circ} \mathrm{C}$ and (b) $\Delta T=20^{\circ} \mathrm{C}$ of temperature changes.

by

$$
\text { Auto }=\sum_{k=0}^{w-1} \frac{\sigma}{\sigma+\Delta \sigma} \operatorname{sech}^{2}\left(\frac{t-k \Delta t}{\sigma+\Delta \sigma}\right)
$$

Using the parameters in Fig. 3, Fig. 4(a) shows the ordinary autocorrelation function with the peak of $w=5$ from the $(5 \times 5,5,0,1)$ CHPC codeword $X_{1}$. Fiber temperature fluctuations cause the multiwavelength pulses in $X_{1}$ to broaden and skew in time. Based on the distorted codeword of $X_{1}$ in Fig. 3(b), Fig. 4(b) shows the distorted autocorrelation function, calculated from (4). It is here assumed that the decoder does not know the amounts of time skew experienced by the distorted codeword. So, the decoder simply realigns the pulses in accordance to the original mark-chip locations of $X_{1}$. As shown in Fig. 4(b), the autocorrelation peak is time-skewed to $24.5 \mathrm{ps}$ and the power (i.e., height) is worsened from 5 to 2.22 .

Also using the parameters in Fig. 3, Fig. 5(a) shows the ordinary cross-correlation function between $X_{1}$ and $X_{2}=$ $[(0,0),(1,2),(2,4),(3,1),(4,3)]$, without fiber temperature fluctuations. It is here assumed that $X_{1}$ arrives at a decoder with the address codeword of $X_{2}$. As shown in Fig. 5(a), the height of the cross-correlation function is approximately equal to 1 . This is because the CHPCs are designed to have $\lambda_{c}=1$. When there exist fiber temperature fluctuations, the distorted codeword of $X_{1}$ in Fig. 3(b) gives the distorted crosscorrelation function (at the decoder of $X_{2}$ ) in Fig. 5(b). The distorted cross-correlation function has a peak power of 0.85 . Comparing to the peak power of 0.51 in the distorted pulses in Fig. 3(b), the maximum cross-correlation function is increased (i.e., worsened) to $\lambda_{c}=1.67$ (i.e., $0.85 / 0.51$ ), even though the CHPCs are designed to have $\lambda_{c}=1$ originally.

Although the distorted cross-correlation function in Fig. 5(b) appears lower than the original one in Fig. 5(a), this does not mean that the impact of MAI is reduced. The autocorrelation 


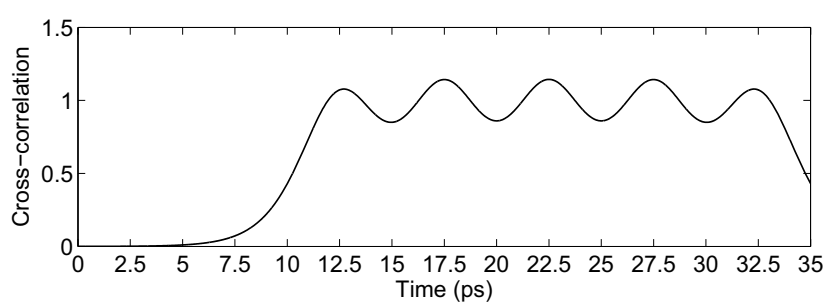

(a)

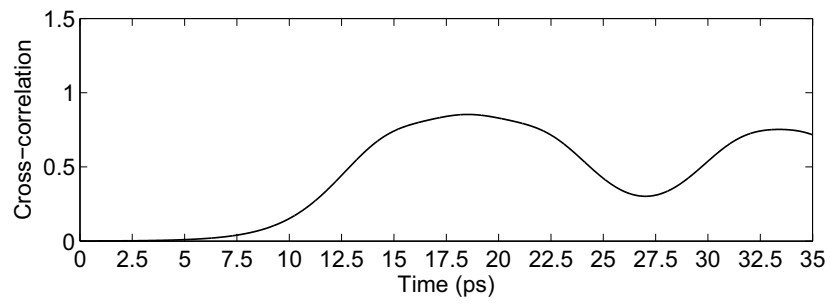

(b)

Fig. 5. Cross-correlation function between $X_{1}$ and $X_{2}=$ $[(0,0),(1,2),(2,4),(3,1),(4,3)]$ of chip-width $t_{c}=5$ ps after $X_{1}$ has traveled in a fiber of $d=20 \mathrm{~km}$ and experienced (a) $\Delta T=0^{\circ} \mathrm{C}$ and (b) $\Delta T=20^{\circ} \mathrm{C}$ of temperature changes.

peak is another important parameter to determine performance because it affects the degree of discrimination against the MAI created by the accumulated cross-correlation function from interfering codewords. As shown in Fig. 4, the distorted autocorrelation peak gets an even larger reduction when compared to the distorted cross-correlation function in Fig. 5. So, the ultimate performance indicator of incoherent O-CDMA is the ratio of the autocorrelation peak to the cross-correlation function. For example, without fiber temperature fluctuations, the original auto-to-cross-correlation ratio is about 5 (i.e., 5/1), as deduced from Figs. 4(a) and 5(a). However, from Figs. 4(b) and 5(b), fiber temperature fluctuations worsen the auto-to-cross-correlation ratio to about 2.61 (i.e., 2.22/0.85). The lowering of this ratio translates into poor performance, as shown in this paper.

In summary, fiber temperature fluctuations also affect the cross-correlation function, not only the autocorrelation function [16]. So, it is important to derive a new performanceanalytical model to more accurately account for the effect of fiber temperature fluctuations - the main theme of this paper.

\section{Relationship to Pulse-Shift Technique}

Lin, et al. [9] introduced the pulse-shift technique to improve the performance of incoherent (asynchronous) OCDMA systems. The technique allows every time slot (or chip) be divided into $g$ sub-chips of equal width, and the pulses in every codeword can randomly be time-shifted to start at any sub-chips in their original chip locations, where $g>1$. Let $\tau_{j}$ be a sub-chip shift of the $j$ th-wavelength pulse, where $\left.\tau_{j} \in\left\{0, t_{c} / g, 2 t_{c} / g, \ldots,(g-1) t_{c} / g\right)\right\}$ for all $j \in\{0,1,2, \ldots, w-1\}$, and $t_{c}$ is the chip-width. The ordered pairs of a shifted copy of codeword $X$ can be denoted as $X^{\prime}=\left[\left(\lambda_{0}, t_{0}+\tau_{0}\right),\left(\lambda_{1}, t_{1}+\tau_{1}\right), \ldots,\left(\lambda_{w-1}, t_{w-1}+\tau_{w-1}\right)\right]$. The analysis in [9] showed that the random pulse-shifts could

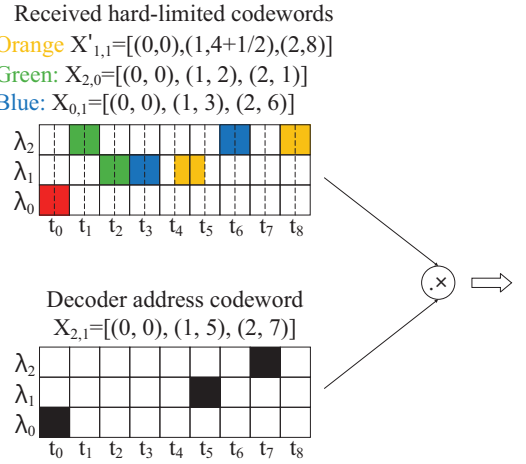

(a)

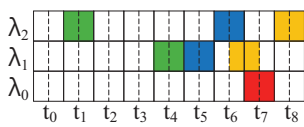

Height $=2$

(b) pulses realigned at $\mathrm{t}_{7}$

Fig. 6. Illustrate how the pulse-shift model of $g=2$ affects the crosscorrelation function: (a) two original codewords $X_{2,0}$ and $X_{0,1}$ and a shifted codeword $X_{1,1}^{\prime}$ arriving at an optical hard-limiting decoder with the address codeword $X_{2,1}$; (b) the maximum cross-correlation function equal to 2 in power but 1.5 in energy at chip $t_{7}$.

be exploited constructively to improve code performance if there existed a feedback mechanism to reconstruct the autocorrelation peak to $w$. As a result, the pulse-shift technique improved code performance as $g$ increased, even though the cross-correlation function was worsened.

Back to the temperature-fluctuation problem on hand, since the pulses of each wavelength are time-shifted by an integral multiple of $\Delta t$, the time-skew effect can equivalently be modeled by subdividing each chip (of chip-width $t_{c}$ ) into subchip of width $\Delta t$, by borrowing the pulse-shift concept in [9]. The chip granularity is here determined by $g=t_{c} / \Delta t$. For example, if $\Delta t=1 \mathrm{ps}$ and $t_{c}=5 \mathrm{ps}$, then this temperaturefluctuation problem can equivalently be classified as the pulseshift model of $g=5$. In Section III, the analytical method in [9] is modified and then applied to more accurately account for the effect of fiber temperature fluctuations in incoherent O-CDMA systems.

\section{Pulse-Power-Detection Model And Analysis}

In this section, the pulse-shift model under pulse-power and pulse-energy detection in incoherent (asynchronous) O-CDMA is studied and analyzed.

Using the $(3 \times 9,3,0,1)$ CHPCs with $L=w=p_{1}=p_{2}=3$ and $N=p_{1} p_{2}=9$ as an example, they have $p_{1} p_{2}=9$ codewords, $X_{i_{1}, i_{2}}=\left[(0,0),\left(1, i_{1}+i_{2} p_{1}\right),\left(2,2 \odot p_{1} i_{1}+(2 \odot\right.\right.$ $\left.\left.\left.p_{2} i_{2}\right) p_{1}\right)\right]$, where $i_{1} \in[0,2]$ and $i_{2} \in[0,2]$. Fig. 6 illustrates the cross-correction process when two original codewords $X_{2,0}=[(0,0),(1,2),(2,1)]$ and $X_{0,1}=[(0,0),(1,3),(2,6)]$ and one shifted codeword $X_{1,1}^{\prime}=[(0,0),(1,4+1 / 2),(2,8)]$ arrive at an optical hard-limiting decoder with the address codeword of $X_{2,1}=[(0,0),(1,5),(2,7)]$. Fig. 6(b) shows that the distorted cross-correlation function has the peak power (or height) of 2 but the energy (or area) of 1.5 in chip $t_{7}$. As illustrated, the pulse-shift creates an undesirable effect to the cross-correlation function.

Fig. 7 illustrates the autocorrection process when the shifted codeword $X_{2,1}^{\prime}=[(0,0),(1,5+1 / 2),(2,7+1 / 2)]$ arrives at an optical hard-limiting decoder with the address codeword of $X_{2,1}=[(0,0),(1,5),(2,7)]$. Fig. $7(\mathrm{~b})$ shows that the distorted 
Received hard-limited codeword Red: $X_{2,1}^{\prime}=[(0,0),(1,5+1 / 2),(2,7+1 / 2)]$

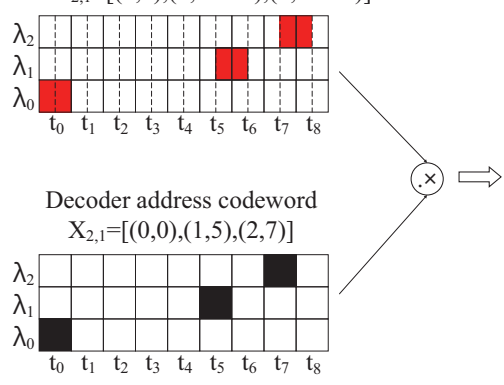

(a)

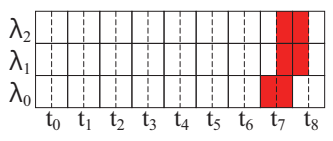

pulses realign at $t_{7}$

Height $=3$

Decode to 1

(b)
Fig. 7. Illustrate how the pulse-shift model of $g=2$ affects the autocorrelation function: (a) a shifted codeword $X_{2,1}^{\prime}=[(0,0),(1,5+1 / 2),(2,7+$ $1 / 2)$ ] arriving at an optical hard-limiting decoder with the address codeword $X_{2,1}=[(0,0),(1,5),(2,7)]$; (b) the autocorrelation peak equal to 3 in power but 2 in energy at chip $t_{7}$.

autocorrelation function has the peak power (or height) of 3 but the energy (or area) of 2 in chip $t_{7}$. For the code weight of $w=3$, the decision-threshold level of the thresholder at the back-end of the optical decoder should normally be set to $T h=w=3$. The unit of $T h$ is measured in terms of energy if the conventional energy (optoelectronic) thresholders are used, but in terms of power if the power (all-optical) thresholders are used. Using the all-optical thresholders, the autocorrelation peak with the power (or height) of 3 in Fig. 7(b) will be recovered as a data bit of 1 correctly. However, if the optoelectronic thresholders are used, the same autocorrelation peak, but with the energy (or area) of 2, will not be detected as a data bit of 1 , thus giving a detection error.

In summary, the pulse-energy detection has a tendency of giving pessimistic performance in the pulse-shift model because of lower autocorrelation-peak energy caused by pulseshifts (e.g., due to fiber temperature fluctuations). On the other hand, the pulse-power detection may still reproduce the autocorrelation-peak power to the original level (e.g., see Fig. 7). Nevertheless, both detection methods in the pulse-shift model see worsening in the cross-correlation function (e.g., see Fig. 6).

\section{A. Analysis of Pulse-Shift, Power-Detection Model}

Since an incoherent O-CDMA system should normally operate in a MAI-limited environment [24], the effect of physical noises is neglected in this section. Also, in OOK, a data bit of 1 cannot be recovered wrongly as a 0 at a decoder because an autocorrelation peak exists on top of MAI. However, a data bit of 0 can be decided wrongly as a 1 if the MAI raises above the autocorrelation peak (i.e., the decision threshold).

In general, the hard-limiting error probability of a family of $\left(L \times N, w, \lambda_{a}, \lambda_{c}\right)$ 2-D optical codes in incoherent (asynchronous) OOK O-CDMA systems is given by [1], [3]

$$
P_{e}=\frac{1}{2} \sum_{k=T h}^{w}\left(\begin{array}{l}
w \\
k
\end{array}\right) \sum_{i=0}^{k}(-1)^{k-i}\left(\begin{array}{l}
k \\
i
\end{array}\right)\left[\sum_{j=0}^{\lambda_{c}} \frac{\left(\begin{array}{c}
i \\
j
\end{array}\right)}{\left(\begin{array}{c}
w \\
j
\end{array}\right)} q_{\lambda_{c}, j}\right]^{K-1}
$$

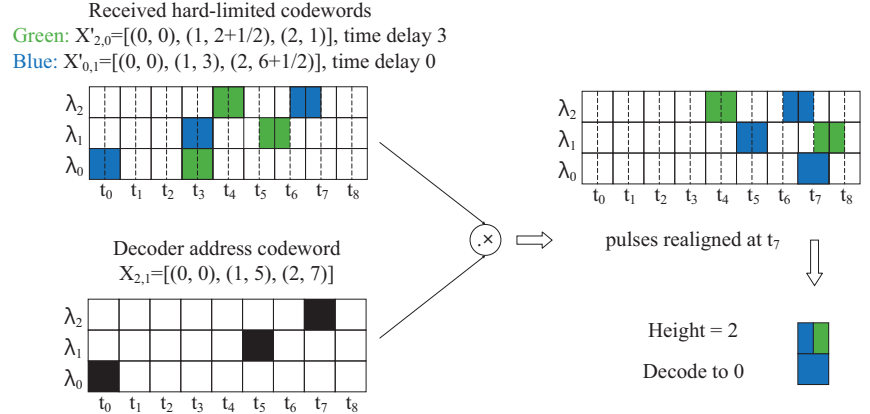

Fig. 8. Cross-correlation process of pulse-shifted interfering codewords $X_{2,0}^{\prime}$ and $X_{0,1}^{\prime}$ arriving at a decoder with the address codeword of $X_{2,1}$.

where the factor $1 / 2$ comes from the assumption of equiprobability of data bits of 0 and $1, T h$ is the decision-threshold level, $K$ represents the number of simultaneous users, and $q_{\lambda_{c}, j}$ denotes the probability of getting $j \in\left[0, \lambda_{c}\right]$ hits in the sampling time of the cross-correlation function. These hit probabilities are generally related by [1], [3], [6]

$$
\begin{aligned}
& \sum_{j=0}^{\lambda_{c}} q_{\lambda_{c}, j}=1 \\
& \sum_{j=0}^{\lambda_{c}} j q_{\lambda_{c}, j}=\frac{w^{2}}{2 L N}
\end{aligned}
$$

For the $(L \times N, w, 0,1)$ 2-D optical codes, such as the CHPCs, the 1-hit probability becomes $q_{1,1}=w^{2} /(2 L N)$ and the 0 -hit probability becomes $q_{1,0}=1-q_{1,1}=1-w^{2} /(2 L N)$ by applying $\lambda_{c}=1$ to (6) and (7).

Without any pulse-shifts, the autocorrelation peak is always equal to $w$ and the decision-threshold level is normally set to $T h=w$ for recovering a data bit of 1 whenever an autocorrelation peak is detected at the thresholder.

Due to the pulse-shift model, the time-shifted pulses from interfering codewords do not always fall entirely into their mark chips. For example, Fig. 8 shows that there exists three interfering pulses stacking up at chip $t_{7}$ in the cross-correlation function of the pulse-shifted interfering codewords $X_{2,0}^{\prime}$ and $X_{0,1}^{\prime}$ at the decoder of $X_{2,1}$, where $g=2$ is assumed. After the correlation process, the pulse of $\lambda_{1}$ from $X_{2,0}^{\prime}$ is found in the second half of chip $t_{7}$ and the first half of chip $t_{8} . X_{0,1}^{\prime}$ contributes the pulse of $\lambda_{0}$ entirely to $t_{7}$, whereas the pulse of $\lambda_{2}$ is found in the second half of $t_{6}$ and the first half of $t_{7}$. As a result, these three pulses create one full hit and two partial hits stacking side-by-side at chip $t_{7}$. They give a combined peak power (or height) of 2, as shown in Fig. 8(b). (Note: The autocorrelation peak should be equal to the code weight $w=3$ if there is no pulse-shift.) In other words, multiple partial hits in one chip do not necessarily result in one full-hit in that chip. Thus, this section applies a new analytical method to account for this situation.

With every chip being divided into $g$ equal-width sub-chips, the new pulse-shift, power-detection model is formulated as follows. Whenever there exists at least one sub-chip in a chip being hit by an interfering pulse in the cross-correlation 
function, this partial hit will first be considered as one "valid" hit. New (valid) hit probabilities, $q_{\lambda_{c}, j, g}$ for $j \in\left[0, \lambda_{c}\right]$, for the $(L \times N, w, 0,1)$ CHPCs are then formulated, where $q_{\lambda_{c}, j, g}$ represent the probability of getting a total of $j \in\left[0, \lambda_{c}\right]$ hits in a chip of the cross-correlation function. Afterward, the actual hit probabilities, $q_{\lambda_{c}, j, g}^{\prime}$, for the pulse-shift, powerdetection model are calculated by scaling down $q_{\lambda_{c}, j, g}$ with some appropriate scaling factors in order to reflect the actual contribution of these partial hits.

Theorem 1: For the $(p \times p, p, 0,1)$ CHPCs, the hit probabilities in the pulse-shift, power-detection model are derived as

$$
\begin{aligned}
& q_{2,0, g}^{\prime}=\frac{6 g^{2}-g-1}{8 g^{2}} \\
& q_{2,1, g}^{\prime}=\frac{g+1}{4 g^{2}} \\
& q_{2,2, g}^{\prime}=\frac{2 g^{2}-g-1}{8 g^{2}}
\end{aligned}
$$

To validate this new analytical model, it is shown in the limiting case of $g=1$ (i.e., no pulse-shift) that these hit probabilities will be degenerated to $q_{2,2,1}^{\prime}=0$ and $q_{2,1,1}^{\prime}=q_{2,0,1}^{\prime}=1 / 2$, corresponding to the existence of one-hits only (i.e., $\lambda_{c}=1$ ). For the case of $\lambda_{c}=1$, it is found that $q_{1,1}=w^{2} /(2 L N)=p^{2} /\left(2 p^{2}\right)=1 / 2$ and $q_{1,0}=1-q_{1,1}=1 / 2$ in the conventional hit-probability model in (6) and (7). Same hit probabilities are found in both models, thus validating the new analytical model.

Proof: See Appendix A.

Theorem 2: For the $\left(p \times p^{2}, p, 0,1\right)$ CHPCs, the hit probabilities in the pulse-shift, power-detection model are derived as

$$
\begin{aligned}
q_{2,0, g}^{\prime} & =\frac{4 g^{3}\left(4 p^{2}+2 p-1\right)-4 g^{2} p(2 p+1)+g-1}{8 g^{2} p(2 g-1)(p+1)} \\
q_{2,1, g}^{\prime} & =\frac{2 g p-p+1}{2 p(p+1)(2 g-1)} \\
q_{2,2, g}^{\prime} & =\frac{(g-1)(2 g+1)}{8 g^{2} p(p+1)}
\end{aligned}
$$

To validate this new analytical model, it is shown in the limiting case of $g=1$ (i.e., no pulse-shift) that the hit probabilities will be degenerated to $q_{2,2,1}^{\prime}=0$ and $q_{2,1,1}^{\prime}=1 /(2 p)$ and $q_{2,0,1}^{\prime}=1-1 /(2 p)$, corresponding to the existence of onehit only (i.e., $\left.\lambda_{c}=1\right)$. For the case of $\lambda_{c}=1$, it is found that $q_{1,1}=w^{2} /(2 L N)=1 /(2 p)$ and $q_{1,0}=1-q_{1,1}=1-1 /(2 p)$ in the conventional hit-probability model in (6) and (7). Same hit probabilities are found in both models, thus validating the new analytical model.

Proof: See Appendix B.

To compute the hard-limiting error probability of the $(L \times$ $N, w, 0,1)$ optical codes, such as the CHPCs, in the pulseshift, power-detection model, the original hit probabilities, $q_{\lambda_{c}, j}$, in (5) are substituted with $q_{\lambda_{c}, j, g}^{\prime}$ in Theorems 1 and 2. As explained in Appendix A, the fixed pulse-shift pattern [i.e, governed by $k \Delta t$ in (3), caused by fiber temperature fluctuations] belongs to a special case of all the possible pulseshift patterns involved in the derivations of the "average" hit probabilities in the theorems. Moreover, the hit probabilities of this fixed pulse-shift pattern are found identical to the "average" hit probabilities. Thus, this pulse-shift model can also be applied to analyze the effect of fiber temperature fluctuations in incoherent O-CDMA systems and complement the deficiency in the analytical model in [16].

\section{B. Analysis of Pulse-Shift, Energy-Detection Model}

In the original pulse-shift model [9], the decision-threshold level was set to $T h=w$ because it was assumed that the decoder consisted of a feedback mechanism to realign the autocorrelation peak to $w$. Because the energy in the crosscorrelation function can be a non-integer (e.g., see Fig. 6), Lin, et al. [9] started with the " $g$-granulated" error-probability equation in [9, eq. (12)] by means of Markov chain and then computed the " $g$-granulated" hit probabilities $q_{k^{\prime}, l^{\prime}, g}$ in [9, eq. (14)], where $k^{\prime}$ and $l^{\prime}$ take the values from the set of $\{0,1 / g, 2 / g, \ldots, 3-2 / g\}$.

In this section, it is assumed that the decoder does not have the pulse-shift information. So, the decision-threshold level cannot simply be set to $T h=w$, and the analysis in [9] is not applicable to this pulse-shift, energy-detection model. Nevertheless, it is noted that [29, eq. (21)] is the general form of [9, eq. (12)] because the former becomes the latter when $n=T h=w$. In the following, the general form of the " $g$ granulated" error probability in [29, eq. (21)] is used to adjust the decision-threshold level under the pulse-energy detection.

Using $w=3$ and $g=2$ as an example, the three pulses in a codeword can be shifted by one sub-chip or no shift in the pulse-shift model. The autocorrelation peak and thus the decision-threshold level $T h$ can range from 1.5 to 3 in energy after realigning these three pulses in the correct-address decoder. Let's denote no shift as a 0 and one sub-chip shift as a 1 to represent the time-shift pattern of the three pulses in a codeword. Then, the ordered triplet $(0,0,0)$ represents that there are no shifts in the three respective pulses in the codeword. This case occurs with a probability of $1 / 8$ and give an autocorrelation peak of 3 (in energy). Similarly, the ordered triplets $(0,0,1),(0,1,0)$, and $(1,0,0)$ have the occurrence probability of $1 / 8$ each and an autocorrelation peak of 2.5 (in energy) (e.g., see Fig. 7). The ordered triplets $(0,1,1)$, $(1,1,0)$, and $(1,0,1)$ have the occurrence probability of $1 / 8$ each and an autocorrelation peak of 2 (in energy). Finally, the ordered triplet $(1,1,1)$ has the occurrence probability of $1 / 8$ and an autocorrelation peak of 1.5 (in energy). However, these autocorrelation-peak (i.e., decision-threshold) values cannot directly be applied to [29, eq. (21)] to compute the error probability for this pulse-shift, energy-detection model because [29, eq. (21)] requires $T h$ be an integer.

By modifying [9, eq. (12)] and [29, eq. (21)], the pulseshift, energy-detection, hard-limiting error probability with the decision-threshold level $T h^{\prime}$ can be formulated as

$$
\begin{aligned}
P_{e, T h^{\prime}}^{\prime}= & \frac{1}{2} \sum_{n=T h^{\prime}}^{w}\left(\begin{array}{c}
w \\
n
\end{array}\right) \sum_{j=0}^{w-n}\left(\begin{array}{c}
w-n \\
j
\end{array}\right) 2^{j} \\
& \times \sum_{i=0}^{2 n+j}(-1)^{2 n+j-i}\left(\begin{array}{c}
2 n+j \\
i
\end{array}\right)
\end{aligned}
$$




$$
\times\left[\sum_{k=0}^{\lceil 3-2 / g\rceil} \sum_{l=0}^{\lceil 3-2 / g\rceil} \frac{\left(\begin{array}{c}
i \\
k+l
\end{array}\right)}{\left(\begin{array}{c}
2 w \\
k+l
\end{array}\right)} Q_{k, l, g}\right]^{K-1}
$$

The hit probabilities, $Q_{k, l, g}$, were derived in [9, eq. (14)], where $k$ and $l$ take the values from the set of $\{0,1,2, \ldots,\lceil 3-$ $2 / g\rceil\}$.

To get around the integer threshold-level restriction of (14), the following steps are here applied:

1) In the pulse-shift, energy-detection model, all the possible autocorrelation-peak (i.e., decision-threshold) values anticipated in the code set in study are averaged to give $T h^{\prime}$.

2) If $T h^{\prime}$ is an integer, it is directly applied to (14) to compute the pulse-shift, energy-detection, hard-limiting error probability.

3) If $T h^{\prime}$ is not an integer, it is rounded down and up to the nearest integers, giving $T h_{\text {floor }}^{\prime}=\left\lfloor T h^{\prime}\right\rfloor$ and $T h_{\text {ceil }}^{\prime}=\left\lceil T h^{\prime}\right\rceil$, respectively. These two integer threshold values are then applied to (14) to find their associated error probabilities $P_{e, T h_{\mathrm{fl}}^{\prime}}^{\prime}$ and $P_{e, T h_{\text {ceil }}^{\prime}}^{\prime}$, respectively. Finally, they are averaged by applying

$$
P_{e, \text { ave }}^{\prime}=P_{e, T h_{\text {floor }}^{\prime}}^{\prime} \times d_{\text {floor }}+P_{e, T h_{\text {ceil }}^{\prime}}^{\prime} \times d_{\text {ceil }}
$$

where the factors $d_{\text {floor }}=1-\left(T h^{\prime}-T h_{\text {floor }}^{\prime}\right)$ and $d_{\text {ceil }}=1-\left(T h_{\text {ceil }}^{\prime}-T h^{\prime}\right)$ represent the distances of $T h_{\text {floor }}^{\prime}$ and $T h_{\text {ceil }}^{\prime}$ from the averaged threshold value $T h^{\prime}$, respectively.

Use the same example with $w=3$ and $g=2$, the averaged threshold value is computed by $T h^{\prime}=[3 \times(1 / 8)+2.5 \times$ $(3 / 8)+2 \times(3 / 8)+1.5 \times(1 / 8)] / 4=2.25$ and then $T h_{\text {ceil }}^{\prime}=$ $\left\lceil T h^{\prime}\right\rceil=3$ and $T h_{\text {floor }}^{\prime}=\left\lfloor T h^{\prime}\right\rfloor=2$. Also, $d_{\text {floor }}=1-$ $\left(T h^{\prime}-T h_{\text {floor }}^{\prime}\right)=0.75$ and $d_{\text {ceil }}=1-\left(T h_{\text {ceil }}^{\prime}-T h^{\prime}\right)=$ 0.25 . Applying these values to (14) and (15), the average hardlimiting error probability of the $(L \times N, w, 0,1)$ optical codes, such as the CHPCs, in the pulse-shift, energy-detection model can be calculated.

The pulse-shift, energy-detection model can complement the deficiency of the energy-detection analytical model in [16]. Osadola, et al. [16] assumed that the cross-correlation function was not affected by the pulse-shifts created by fiber temperature fluctuations. However, as shown in Section II, this assumption is not true. This energy-detection model can give a more realistic performance than that in [16].

\section{NumericAl Results AND VALIDATION}

Figs. 9-11 compare the hard-limiting error probabilities, $P_{e}$ of (5) and $P_{e \text {,ave }}^{\prime}$ of (15), of the $(L \times N, w, 0,1)$ CHPCs in the pulse-shift, power-detection and energy-detection models for chip granularity of $g=2,3$, and 4, respectively, where $L=w=p=\{3,5,7\}$ and $N=p^{2}=\{9,25,49\}$. The error probabilities of the power-detection model are computed by applying the hit probabilities in (11)-(13) to the $P_{e}$ equation of (5). In the power detection with pulse-shifts, the autocorrelation peak is equal to $w$ because the pulses will not be shifted out of the entire chip (e.g., see Fig. 7), thus $T h=w$ in (5). The error probabilities of the energy-detection model are

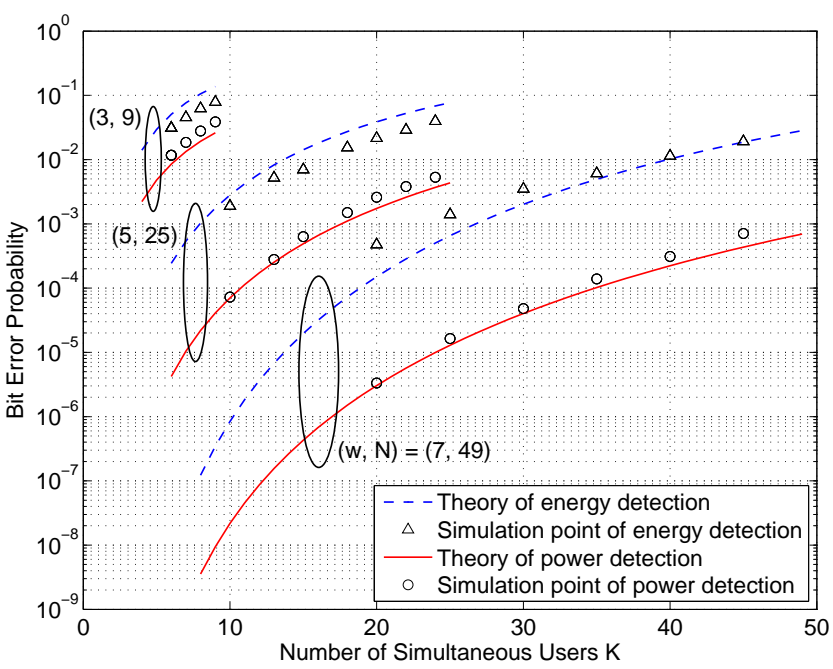

Fig. 9. Hard-limiting error probabilities versus the number of simultaneous users $K$ for the $(L \times N, w, 0,1)$ CHPCs and $g=2$ in the pulse-shift, power-detection and energy-detection models, where $L=w=\{3,5,7\}$ and $N=\{9,25,49\}$.

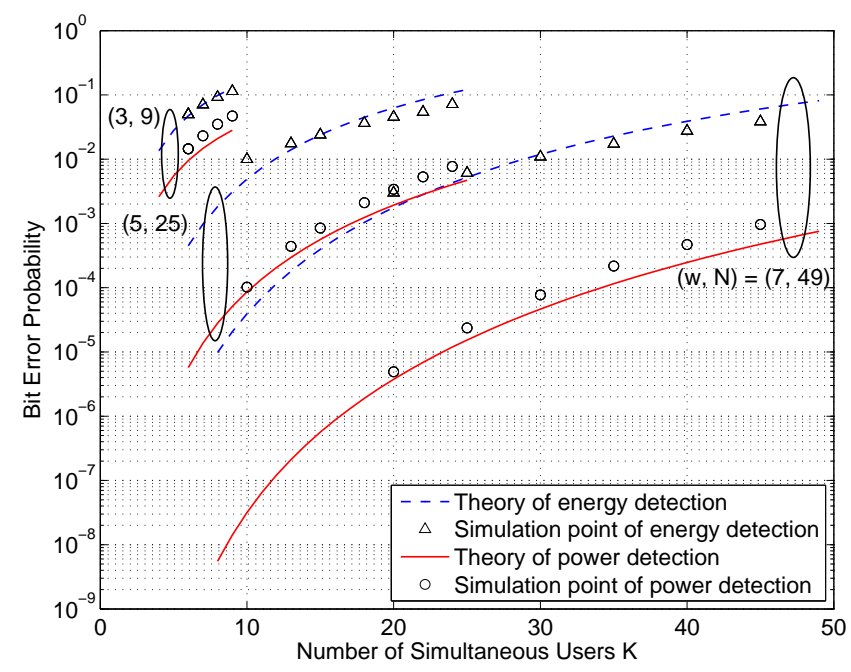

Fig. 10. Hard-limiting error probabilities versus the number of simultaneous users $K$ for the $(L \times N, w, 0,1)$ CHPCs and $g=3$ in the pulse-shift, power-detection and energy-detection models, where $L=w=\{3,5,7\}$ and $N=\{9,25,49\}$.

obtained by following the steps in the computation of $P_{e, \text { ave }}^{\prime}$ in (15). In general, the performance (i.e., error probability) gets worse as $K$ increases, due to stronger MAI. The performance improves with $L, N$, or $w$, for both detection models. This is because the increment of $L$ or $N$ reduces the hit probabilities, and the increment of $w$ increases the power and energy of the autocorrelation peaks. Given a set of $(w, N), g$, and $K$ values, the solid curves of the power-detection model are always lower (i.e., better $P_{e}$ ) than the dashed curves of the energydetection model. This is because the former have less chances of recovering wrong data bits, as explained in Section III. Computer simulations are also performed and the simulation results are shown as the "o" and " $\triangle$ " symbols in the figures. 


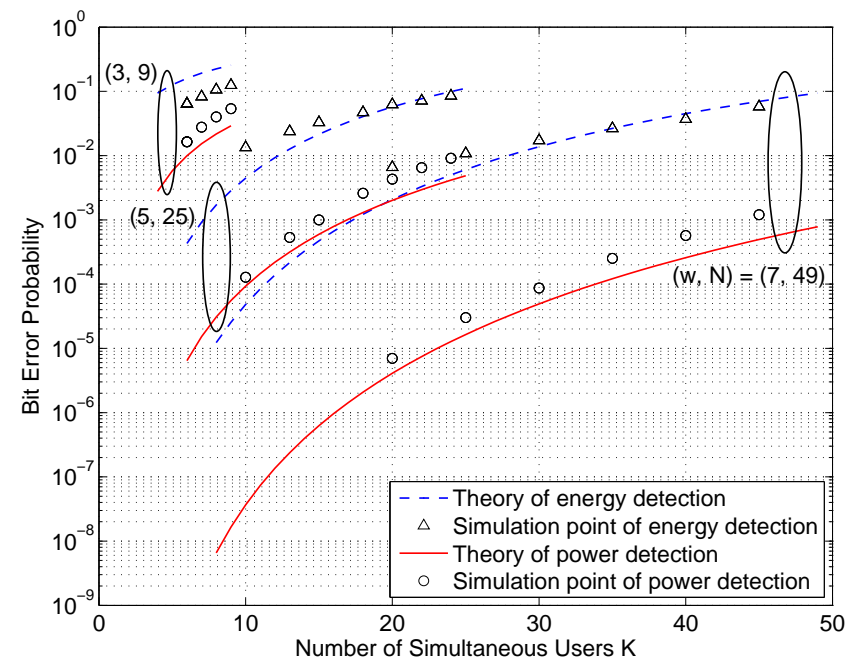

Fig. 11. Hard-limiting error probabilities versus the number of simultaneous users $K$ for the $(L \times N, w, 0,1) \mathrm{CHPCs}$ and $g=4$ in the pulse-shift, power-detection and energy-detection models, where $L=w=\{3,5,7\}$ and $N=\{9,25,49\}$.

The simulation symbols match with the analytical curves, thus validating the new analytical models in Section III.

The computer simulations are based on the so-called MonteCarlo simulation method. They begin with randomly assigning one distinct codeword to each user (or receiver) as its address signature. Each of the $K$ simultaneous users transmits the address codeword of its intended receiver when a data bit of 1 is conveyed. The time frame of the fiber-optic channel is divided into $N$ chips per bit period, and any simultaneous user can start to transmit its codeword at any chip to simulate the bit asynchronism in incoherent (asynchronous) O-CDMA. The multiwavelength pulses in every codeword are time-shifted randomly and independently to any one of the $g$ sub-chips in the original chip positions of the pulses. In the hard-limiting receiver, if there are multiple pulses of the same wavelength (coming from multiple interfering codewords) arriving at the same chip, these pulses are hard-limited and only counted as one pulse (of that wavelength) at that chip. Afterward, correlation is performed by comparing the wavelengths in all $w$ mark chips of the address codeword of the receiver with the wavelengths and chip locations of the pulses of the hardlimited signal. If there exists a pulse with the wavelength and chip location matching one of the mark chips of the address codeword, "one hit" is added to the count. Due to the timeshifts, the hit can occur in one or several consecutive sub-chips within a mark chip of the address codeword and is called a "partial" hit. In the energy detection, the total energy (or area) of the partial and full hits at the $w$ mark chips of the address codeword are summed up. A data bit of 1 is recovered whenever the total energy in a chip is as high as the decisionthreshold level Th. On the other hand, the power detection sums up the total power (i.e., height) of the hits (sub-chip by sub-chip) at the $w$ mark chips of the address codeword. A data bit of 1 is recovered whenever the total power in one sub-chip is as high as $T h$. To determine the proper $T h$

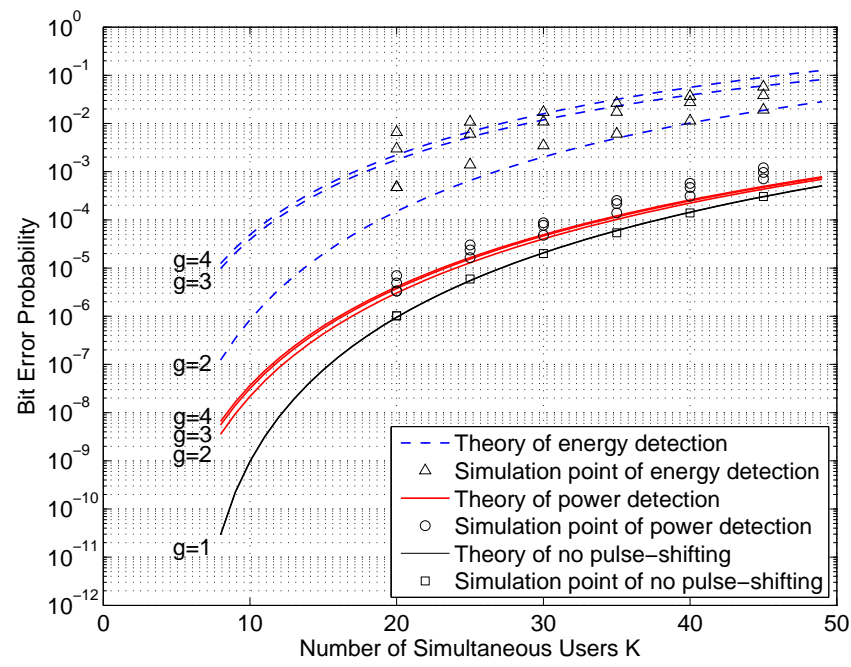

Fig. 12. Hard-limiting error probabilities versus the number of simultaneous users $K$ for the $(7 \times 49,7,0,1) \mathrm{CHPCs}$ and $g=\{1,2,3,4\}$ in the pulseshift, power-detection and energy-detection models.

value in each simulation iteration, a training codeword (with random time-shifts among its pulses), which corresponds to the address of the intended receiver, will be transmitted before the actual data-bit transmission. This training codeword gives an autocorrelation peak but can be lowered, due to the pulseshifts. The energy and power of this peak will be used as the decision-threshold level for energy and power detection, respectively, in that simulation iteration. For the case of a codeword arriving at its intended receiver, the autocorrelation peak, which is equal to $T h$, will lead to the recovery of a data bit of 1 correctly. If the accumulated cross-correlation function at the expected chip-location of the autocorrelation peak is as high as $T h$, a data bit of 1 will be recovered wrongly. The number of decision errors is counted by comparing the recovered data-bit sequence with the transmitted data-bit sequence. Finally, the error probability is calculated as the ratio of the number of decision errors to the total number of transmitted data bits. Moreover, the number of data bits (i.e., simulation sample size) used for each given number of simultaneous users $K$ in the computer simulation is 100 times of the reciprocal of the targeting error probability in order to provide sufficient simulation iterations.

Fig. 12 compares the hard-limiting error probabilities, $P_{e}$ of (5) and $P_{e, \text { ave }}^{\prime}$ of $(15)$, of the $(7 \times 49,7,0,1)$ CHPCs in the pulse-shift, power-detection and energy-detection models as a function of chip granularity $g=\{1,2,3,4\}$. The case of $g=1$ corresponds to the original codewords with no pulseshifts. The methods to determine the $T h$ values, calculate the error probabilities, and perform the computer simulations are identical to those of Figs. 9-11. In general, the performance gets worse as $g$ increases because a large $g$-value increases the number of possible sub-chip positions for pulse-shifts. So, the probability of getting the partial hits increases with $g$. As shown in the theoretical curves and simulation symbols of $g=\{2,3,4\}$, it is important to note that the powerdetection model can effectively reduce the deleterious effect 
of pulse-shifts (e.g., due to fiber temperature fluctuations) as $P_{e}$ is a weak function of $g$. As the choice of the $g$ value is governed by the degree of time-skew severity caused by fiber temperature fluctuations, one benefit of the new pulse-shift, power-detection model is on the insensitivity to this effect.

Moreover, the results from the energy-detection model (i.e., the dashed curves) in Fig. 12 are opposite to those in [9]. A feedback mechanism in the decoder was assumed in [9] in order to track and compensate for the pulse-shifts so that the original autocorrelation peak was reconstructed. As a result, the performance was shown to improve with $g$ in [9]. On the other hand, the pulse-shift, energy-detection model assumes that no feedback mechanism is available in the decoder. So, the performance gets worse as $g$ increases.

\section{CONCLUSION}

In this paper, the effect of fiber temperature fluctuations to the autocorrelation and cross-correlation functions of 2D wavelength-time codes, such as the CHPCs, in incoherent (asynchronous) O-CDMA systems were investigated. Modifying the pulse-shift technique in [9], the time skews of the multiwavelength pulses in codewords were modeled as a function of chip granularity. Furthermore, the effects of power and energy detection to the autocorrelation peak, crosscorrelation function, and the decision-threshold level were studied. New performance analyses for the pulse-shift, powerdetection and energy-detection models were formulated and validated by computer simulations. Our study showed that the power-detection model gave more realistic and better performance than the conventional energy-detection model. The power-detection model could effectively reduce the effect of fiber temperature fluctuations as the performance was a weak function of chip granularity, thus less sensitive to the time skew.

\section{APPENDIX A}

\section{PROOF OF THEOREM 1}

For the $(p \times p, p, 0,1)$ CHPCs, the 0-hit, 1-hit, and 2-hit probabilities can be derived as

$$
\begin{aligned}
& q_{2,0, g}=\frac{1}{2} \\
& q_{2,1, g}=\frac{1}{2} \times \frac{\text { number of 1-hits }}{\text { total number of hits }}=\frac{1}{2 g} \\
& q_{2,2, g}=1-q_{2,0, g}-q_{2,1, g}=\frac{g-1}{2 g}
\end{aligned}
$$

respectively. Because of the code structure of the CHPCs, an interfering codeword can always create one 1-hit in the crosscorrelation function. Due to equiprobability of data bits of 0 and 1 , the probability of getting 0 -hits, $q_{2,0, g}$ in (A.1), is then equal to $1 / 2$ (from the data bits of 0 only).

In general, the $(L \times N, w, 0,1)$ CHPCs have $\phi=N$ codewords. To calculate the total number of possible hits, one codeword is chosen to correlate with the other $\phi-1$ codewords. As each pulse can be shifted to start at one of the $g$ sub-chips in a mark chip, there are $g^{w}$ possible starting locations for the $w$ pulses in each codeword. Also, a codeword can have up to $N$ cyclic-shifts due to bit asynchronism in incoherent O-CDMA. Thus, the total number of possible hits is found to be $\phi(\phi-1) g^{w} N$. For the $(p \times p, p, 0,1)$ CHPCs, the total number of possible hits becomes $p(p-1) g^{p} p$. Due to the code structure, an interfering codeword always creates one 1-hit in the cross-correlation function. If there exists another pulse being shifted (even by one sub-chip), this will cause a total of two (partial or full) 1-hits in one chip. To prevent this kind of "2-hits" from occurring, the $g^{w}$ possible starting locations for the $w$ pulses in each codeword need to be reduced to $g^{w-1}$. Then, the number of 1-hits in the $(p \times p, p, 0,1)$ CHPCs can be found to be $\phi(\phi-1) g^{w-1} N=p(p-1) g^{p-1} p$. So, the ratio of "the number of 1-hits" to "the total number of all possible hits" becomes $1 / g$, and the final form of (A.2) is resulted.

Due to the time-shifts, sometimes the pulses of an interfering codeword cannot stack up atop each other in the cross-correlation function but side-by-side, instead. In other words, the contribution of the power of each shifted pulse may not be complete; an alternative method is needed to calculate the hit probabilities for these partial hits. For the $(p \times p, p, 0,1)$ CHPCs, two scaling factors, $(g+1) /(2 g)$ and $(2 g+1) /(4 g)$, are used to scale down $q_{\lambda_{c}, j, g}$ in order to reflect the actual (power) contribution of these partial hits. The scaled hit probabilities are given by

$$
\begin{aligned}
& q_{2,0, g}^{\prime}=1-q_{2,2, g}^{\prime}-q_{2,1, g}^{\prime} \\
& q_{2,1, g}^{\prime}=q_{2,1, g} \times \frac{g+1}{2 g}=\left(\frac{1}{2 g}\right)\left(\frac{g+1}{2 g}\right) \\
& q_{2,2, g}^{\prime}=q_{2,2, g} \times \frac{2 g+1}{4 g}=\left(\frac{g-1}{2 g}\right)\left(\frac{2 g+1}{4 g}\right)
\end{aligned}
$$

After some manipulations, the final form of (8)-(10) in Theorem 1 are formulated for the pulse-shift, power-detection model.

The scaling factors, $(g+1) /(2 g)$ and $(2 g+1) /(4 g)$, are derived by counting all possible patterns of the partial hits in the cross-correlation functions of the $(p \times p, p, 0,1)$ CHPCs. For example, for the case of $g=2$, there are two sub-chips per chip. Let's denote "xy" as the hit pattern of the partial hits in the first and second sub-chips, respectively, where $x$ and $y \in\{0,1,2\}$, in which " 0 " means no hit, " 1 " means one partial hit, and " 2 " means two partial hits in the corresponding subchip. Considering all cases of $g=2$ and any $p$ in the pulseshift, power-detection model, each of the (partial or full) hits contributed by the two hit-patterns " 01 " and " 11 " is counted as one full hit, and each of the (partial or full) hits contributed by the two hit-patterns " 11 " and " 21 " is counted as two full hits. (Note: the hit-pattern " 11 " comes from " 01 "+" 10 " and the hit-pattern " 21 " comes from "10"+"11.") Afterward, the occurrence probability of each hit-pattern and its expected value are calculated. For the $(p \times p, p, 0,1)$ CHPCs with $g=2$, the occurrence probabilities of the two hit-patterns contributing to the 1 -hits are both equal to $1 / 2$. So, the expected value becomes $(1 / 2) \times(1 / 2)+(2 / 2) \times(1 / 2)=3 / 4$, which can equivalently be written as $(g+1) /(2 g)$ due to $g=2$. Similarly, the occurrence probabilities of the two hit-patterns contributing to the 2-hits are both equal to $1 / 2$. So, the expected value becomes $(2 / 4) \times(1 / 2)+(3 / 4) \times(1 / 2)=5 / 8$, which can 
equivalently be written as $(2 g+1) /(4 g)$ due to $g=2$.

Considering all cases of $g=3$ and any $p$ in the pulseshift, power-detection model, each of the (partial or full) hits contributed by the three hit-patterns "001," "011," and " 111 " is counted as one full hit, and each of the (partial or full) hits contributed by the five hit-patterns " $211, "$ "221," " 101 ," " 121 ," and " 111 " is counted as two full hits. The occurrence probabilities of the three hit-patterns contributing to the 1hits are all equal to $1 / 3$. So, the expected value becomes $(1 / 3) \times(1 / 3)+(2 / 3) \times(1 / 3)+(3 / 3) \times(1 / 3)=2 / 3$, which can equivalently be written as $(g+1) /(2 g)$ due to $g=3$. The occurrence probabilities of the five hit-patterns contributing to the 2 -hits are equal to $1 / 6,1 / 6,1 / 6,1 / 6$, and $1 / 3$, respectively. So, the expected value becomes $(4 / 6) \times(1 / 6)+(5 / 6) \times$ $(1 / 6)+(2 / 6) \times(1 / 6)+(4 / 6) \times(1 / 6)+(3 / 6) \times(1 / 3)=7 / 12$, which can equivalently be written as $(2 g+1) /(4 g)$ due to $g=3$.

According to these results, the general formula of the expected values are given by $(g+1) /(2 g)$ for the 1-hits and $(2 g+1) /(4 g)$ for the 2-hits. These expected values represent the actual power contributions of the partial hits in a chip of the cross-correlation function. They are then used to scale down the hit probabilities, $q_{2,1, g}$ in (A.2) and $q_{2,2, g}$ in (A.3), in order to obtain the final hit-probabilities $q_{2,1, g}^{\prime}$ in (A.5) and $q_{2,2, g}^{\prime}$ in (A.6), respectively, for the pulse-shift, power-detection model.

According to the above derivations, the hit probabilities in (A.1)-(A.3) are computed by considering all possible pulseshift patterns in the codewords. The fixed pulse-shift pattern, governed by $k \Delta t$ in (3) due to fiber temperature fluctuations, belongs to one of these pulse-shift patterns. Thus, the total number of possible hits seen by this fixed pulse-shift pattern is found to be $\phi(\phi-1) g^{w} N / g^{w}=\phi(\phi-1) N$, and the number of 1-hits is $\phi(\phi-1) g^{w-1} N / g^{w}=\phi(\phi-1) N / g$. According to the definition of (A.2), the 1-hit probability of this fixed pulse-shift pattern can be written as $q_{2,1, g}=(1 / 2) \times[\phi(\phi-1) N] /[\phi(\phi-$ $1) N / g]=1 /(2 g)$ for the $(p \times p, p, 0,1)$ CHPCs. So, the general formula of $q_{2,1, g}$ in (A.2) is applicable to this fixed pulse-shift pattern. Following the rest of the appendix, the hit probabilities $q_{2,0, g}$ and $q_{2,2, g}$ and the scaling factors derived in (A.5) and (A.6) are also applicable to this fixed pulse-shift pattern. As a result, the analytical model and numerical results in this paper can be used to account for the effect of fiber temperature fluctuations in incoherent O-CDMA systems.

\section{APPENDIX B \\ PROOF OF THEOREM 2}

According to (A.3), the 2-hit probability of the $(p \times p, p, 0,1)$ $\mathrm{CHPCs}$ can also be written as

$$
q_{2,2, g}=\frac{1}{2} \times \frac{\text { number of } 2 \text {-hits }}{\text { total number of hits }}=\frac{g-1}{2 g}
$$

Thus, the total number of 2-hits is equal to $[(g-1) /(2 g)] \times$ $2 \times$ total number of hits $=(g-1) /(2 g) \times 2 \times \phi(\phi-1) g^{w} N$, where $\phi=w=L=N=p$ in the $(p \times p, p, 0,1)$ CHPCs. This equation can further be rearranged as $p[(g-1) /(2 g) \times 2 p(p-$ 1) $\left.g^{p}\right]$, in which the terms in the square brackets correspond to the number of 2-hits in each codeword.

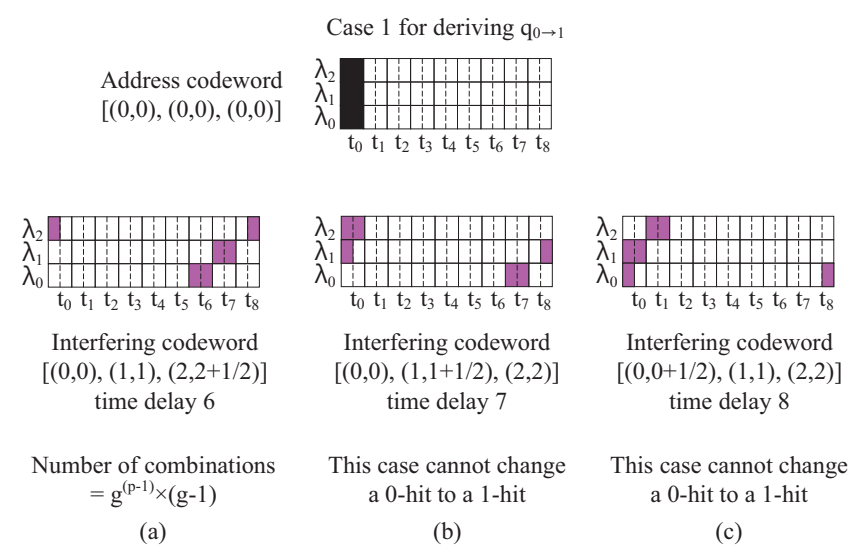

Fig. 13. Illustrate how a pulse of an interfering codeword can change a 0-hit to a 1-hit in the cross-correlation function of the $(3 \times 9,3,0,1)$ CHPCs with $g=2$, in the pulse-shift model for Case 1.

By inspection, it is found that the $(p \times p, p, 0,1)$ and $(p \times$ $\left.p^{2}, p, 0,1\right)$ CHPCs have the same number of 2-hits in each codeword for the same $g$. Thus, the total number of 2-hits in the $\left(p \times p^{2}, p, 0,1\right)$ CHPCs can be represented as $p^{2}[(g-$ $\left.1) /(2 g) \times 2 p(p-1) g^{p}\right]$. The 2-hit probability can then be derived as

$$
\begin{aligned}
q_{2,2, g} & =\frac{1}{2} \times \frac{\text { number of 2-hits }}{\text { total number of hits }} \\
& =\frac{1}{2} \times \frac{p^{2}\left(\frac{g-1}{2 g}\right) 2 p(p-1) g^{p}}{p^{2}\left(p^{2}-1\right) g^{p} p^{2}} \\
& =\frac{(g-1)}{2 g p(p+1)}
\end{aligned}
$$

The 0-hit probability of the $\left(p \times p^{2}, p, 0,1\right)$ CHPCs is formulated by

$$
q_{2,0, g}=q_{0}-q_{0 \rightarrow 1}=1-q_{1}-q_{0 \rightarrow 1}
$$

where $q_{0}$ represents the probability of getting 0-hits in the cross-correlation function in the original case (i.e., no pulseshifts), and $q_{0 \rightarrow 1}$ represents the probability of changing a 0 hit to a 1-hit (caused by pulse-shifts) in the cross-correlation function. There are three pulse-shift cases causing a 0-hit to become a 1-hit, as follows.

Use the $(3 \times 9,3,0,1)$ CHPCs with $g=2$ as an example. Illustrated in Fig. 13(a) for Case 1, the pulse of wavelength $\lambda_{2}$ at chip $t_{8}$ has $\{1 / g, 2 / g, \ldots,(g-1) / g\}$ pulse-shift possibilities of changing a 0 -hit to a 1-hit in the cross-correlation function with the pulse of $\lambda_{2}$ in the address codeword at $t_{0}$. The other $w-1=2$ pulses have $\{0,1 / g, \ldots,(g-1) / g\}$ pulse-shift possibilities of changing a 0 -hit to a 1-hit. The number of pulse-shift combinations in this scenario is found to be $g^{(p-1)}(g-1)=2^{(3-1)}(2-1)=4$, for example, coming from the shifted codewords $[(0,0),(1,1),(2,2+1 / 2)],[(0,0+$ $1 / 2),(1,1),(2,2+1 / 2)],[(0,0),(1,1+1 / 2),(2,2+1 / 2)]$, and $[(0,0+1 / 2),(1,1+1 / 2),(2,2+1 / 2)]$. However, in Fig. 13(b), because the pulse of $\lambda_{2}$ at $t_{0}$ has generated a 1-hit already, the pulse of $\lambda_{1}$ cannot change a 0 -hit to a 1-hit anymore, no matter how much the pulse is shifted. The situation of Fig. 13(c) is 
Address codeword $[(0,0),(0,0),(0,0)]$
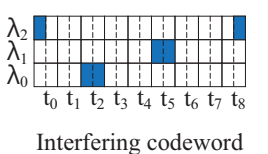

$[(0,0),(1,3),(2,6+1 / 2)]$ time delay 2

Number of combinations $=\mathrm{g}^{(\mathrm{p}-1)} \times(\mathrm{g}-1)$

(a)
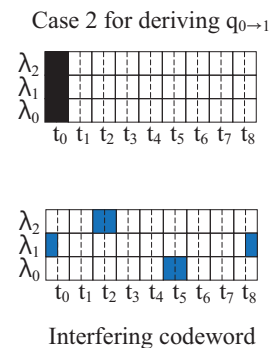

$[(0,0),(1,3+1 / 2),(2,6)]$ time delay 5

Number of combinations $=g^{(p-1)} \times(g-1)$

(b)

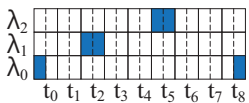

Interfering codeword $[(0,0+1 / 2),(1,3),(2,6)]$ time delay 8

Number of combinations $=\mathrm{g}^{(\mathrm{p}-1)} \times(\mathrm{g}-1)$

(c)

Fig. 14. Illustrate how a pulse of an interfering codeword can change a 0-hit to a 1-hit in the cross-correlation function of the $(3 \times 9,3,0,1)$ CHPCs with $g=2$, in the pulse-shift model for Case 2 .

similar to (b) because the pulse of $\lambda_{0}$ cannot change a 0 hit to a 1-hit, either. In summary, the number of pulse-shift combinations that can change a 0 -hit to a 1-hit in Case 1 is found to be $g^{(p-1)}(g-1) \times 1=4$, where the factor " 1 " means that there is only one wavelength, $\lambda_{2}$, changing a 0 -hit to a 1-hit. Finally, the total number of pulse-shift combinations in Case 1 becomes $g^{(p-1)}(g-1) \times(p-1) p^{2}$ because this case appears $(p-1) p^{2}$ times in the cross-correlation functions of the $\left(p \times p^{2}, p, 0,1\right)$ CHPCs, by inspection.

Illustrated in Fig. 14 for Case 2, the pulse of wavelength $\lambda_{2}$ at chip $t_{8}$ has $\{1 / g, 2 / g, \ldots,(g-1) / g\}$ pulse-shift possibilities of changing a 0 -hit to a 1-hit in the cross-correlation function with the pulse of $\lambda_{2}$ in the address codeword at $t_{0}$. The other $w-1=2$ pulses have $\{0,1 / g, \ldots,(g-1) / g\}$ pulse-shift possibilities of changing a 0 -hit to a 1-hit. The number of pulse-shift combinations in this scenario is found to be $g^{(p-1)}(p-1)=2^{(3-1)}(2-1)=4$, for example, coming from the shifted codewords $[(0,0),(1,3),(2,6+1 / 2)],[(0,0+$ $1 / 2),(1,3),(2,6+1 / 2)],[(0,0),(1,3+1 / 2),(2,6+1 / 2)]$, and $[(0,0+1 / 2),(1,3+1 / 2),(2,6+1 / 2)]$. Different from Case 1, Fig. 14(b) and (c) in Case 2 can also change a 0hit to a 1-hit, and their numbers of pulse-shift combinations are identical to that of (a). In summary, the number of pulseshift combinations that change a 0 -hit to a 1-hit in Case 2 is found to be $g^{(p-1)}(g-1) \times p=4 \times 3=12$, where the factor " $p$ " means that there are $p=3$ wavelengths, $\lambda_{0}, \lambda_{1}$, and $\lambda_{2}$, changing a 0 -hit to a 1-hit. Finally, the total number of pulse-shift combinations in Case 2 becomes $g^{(p-1)}(g-1) p \times(p-1)^{2} p^{2}$ because this case appears $(p-1)^{2} p^{2}$ times in the cross-correlation functions of the $\left(p \times p^{2}, p, 0,1\right)$ CHPCs, by inspection.

Illustrated in Fig. 15(b) for Case 3, the pulse of wavelength $\lambda_{1}$ at chip $t_{8}$ has $\{1 / g, 2 / g, \ldots,(g-1) / g\}$ pulse-shift possibilities of changing a 0 -hit to a 1 -hit in the cross-correlation function with the pulse of $\lambda_{1}$ in the address codeword at $t_{0}$. The other $w-1=2$ pulses have $\{0,1 / g, \ldots,(g-1) / g\}$ pulse-shift possibilities of changing a 0 -hit to a 1-hit. The number of pulse-shift combinations in this scenario is found to be $g^{(p-1)}(p-1)=2^{(3-1)}(2-1)=4$, for example, coming

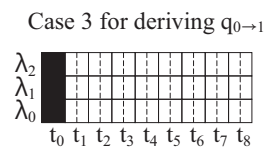

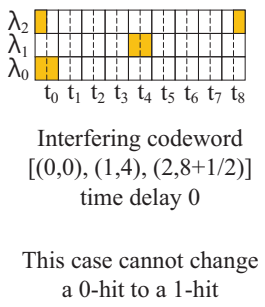

(a)

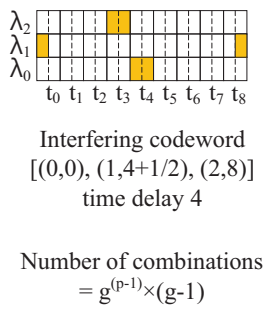

(b)

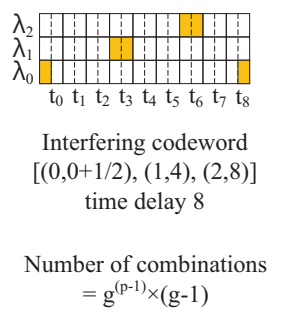

(c)
Fig. 15. Illustrate how a pulse of an interfering codeword can change a 0-hit to a 1-hit in the cross-correlation function of the $(3 \times 9,3,0,1)$ CHPCs with $g=2$, in the pulse-shift model for Case 3 .

from the shifted codewords $[(0,0),(1,4+1 / 2),(2,8)],[(0,0+$ $1 / 2),(1,4+1 / 2),(2,8)],[(0,0),(1,4+1 / 2),(2,8+1 / 2)]$, and $[(0,0+1 / 2),(1,4+1 / 2),(2,8+1 / 2)]$. Also in Fig. 15(c) for Case 3 , the pulse of $\lambda_{0}$ at $t_{8}$ has $\{1 / g, 2 / g, \ldots,(g-1) / g\}$ pulse-shift possibilities of changing a 0-hit to a 1-hit in the cross-correlation function with the pulse of $\lambda_{0}$ in the address codeword at $t_{0}$. The other $w-1=2$ pulses have $\{0,1 / g, \ldots,(g-1) / g\}$ pulse-shift possibilities of changing a 0 -hit to a 1-hit. The number of pulse-shift combinations in this scenario is found to be $g^{(p-1)}(g-1)=2^{(3-1)}(2-$ $1)=4$, for example, coming from the shifted codewords $[(0,0+1 / 2),(1,4),(2,8)],[(0,0+1 / 2),(1,4+1 / 2),(2,8)]$, $[(0,0+1 / 2),(1,4),(2,8+1 / 2)]$, and $[(0,0+1 / 2),(1,4+$ $1 / 2),(2,8+1 / 2)]$. However, in Fig. 15(a), because the pulse of $\lambda_{0}$ at $t_{0}$ has generated a 1-hit already, the two pulses of other wavelengths cannot change a 0-hit to a 1-hit anymore, no matter how much the pulses are shifted. In summary, the number of pulse-shift combinations that change a 0 -hit to a 1hit in Case 3 is found to be $g^{(p-1)}(g-1) \times(p-1)=4 \times 2=8$, where the factor " $p-1$ " means that there are $p-1=2$ wavelengths, $\lambda_{0}$ and $\lambda_{1}$, changing a 0 -hit to a 1-hit. Finally, the total number of pulse-shift combinations in Case 3 becomes $g^{(p-1)}(g-1)(p-1) \times(p-1) p^{2}$ because this case appears $(p-1) p^{2}$ times in the cross-correlation functions of the $\left(p \times p^{2}, p, 0,1\right)$ CHPCs, by inspection.

Combining these three cases, the probability of changing a 0-hit to a 1-hit can be formulated as

$$
\begin{aligned}
q_{0 \rightarrow 1}= & \frac{1}{2} \times \frac{\text { case } 1+\text { case } 2+\text { case } 3}{\text { total number of hits }} \\
= & \frac{g^{(p-1)}(g-1)(p-1) p^{2}}{2 p^{2}\left(p^{2}-1\right) g^{p} p^{2}} \\
& +\frac{g^{(p-1)}(g-1) p(p-1)^{2} p^{2}}{2 p^{2}\left(p^{2}-1\right) g^{p} p^{2}} \\
& +\frac{g^{(p-1)}(g-1)(p-1)(p-1) p^{2}}{2 p^{2}\left(p^{2}-1\right) g^{p} p^{2}} \\
= & \frac{g-1}{2(p+1) g}
\end{aligned}
$$

Thus, the probability of getting 0-hits, defined in (B.3), in the 
$(3 \times 9,3,0,1)$ CHPCs can be derived as

$$
\begin{aligned}
q_{2,0, g} & =1-\frac{w^{2}}{2 L N}-\frac{g-1}{2(p+1) g} \\
& =\frac{2 p-1}{2 p}-\frac{g-1}{2(p+1) g}
\end{aligned}
$$

Furthermore, the probability of getting 1-hits in the $(3 \times$ $9,3,0,1)$ CHPCs can be derived as

$$
q_{2,1, g}=1-q_{2,0, g}-q_{2,2, g}=\frac{2 g p-p+1}{2 g p(p+1)}
$$

Similar to the explanations in Appendix A, for the $(p \times$ $\left.p^{2}, p, 0,1\right)$ CHPCs, two scaling factors, $g /(2 g-1)$ and $(2 g+$ 1) $/(4 g)$, are used to scale down $q_{\lambda_{c}, j, g}$ in order to reflect the actual (power) contribution of these partial hits. The scaled hit probabilities are given by

$$
\begin{aligned}
q_{2,0, g}^{\prime} & =1-q_{s_{2,1, g}}-q_{s_{2,2, g}} \\
q_{2,1, g}^{\prime} & =q_{2,1, g} \times \frac{g}{2 g-1} \\
q_{2,2, g}^{\prime} & =q_{2,2, g} \times \frac{2 g+1}{4 g}
\end{aligned}
$$

After some manipulations, the final form of (11)-(13) in Theorem 2 are formulated for the pulse-shift, power-detection model.

The scaling factors, $g /(2 g-1)$ and $(2 g+1) /(4 g)$, are derived by counting all possible patterns of the partial hits in the cross-correlation functions of the $\left(p \times p^{2}, p, 0,1\right)$ CHPCs. Similar to Appendix A, considering all cases of $g=2$ and any $p$ in the pulse-shift, power-detection model, each of the (partial or full) hits contributed by the three hit-patterns " 01 ," " 10 ," and " 11 " is counted as one full hit, and each of the (partial or full) hits contributed by the two hit-patterns "11" and " 21 " is counted as two full hits. (Note: the hit-pattern " 11 " comes from " 01 " +10 " and the hit-pattern " 21 " comes from "10"+"11.") Afterward, the occurrence probability of each hitpattern and its expected value are calculated. For example of the $(3 \times 9,3,0,1)$ CHPCs with $g=2$, the occurrence probabilities of the three hit-patterns contributing to the 1-hits are equal to $7 / 20,3 / 10$, and $7 / 20$, respectively. So, the expected value becomes $(1 / 2) \times(7 / 20)+(1 / 2) \times(3 / 10)+(2 / 2) \times(7 / 20)=$ $2 / 3$, which can equivalently be written as $g /(2 g-1)$ due to $g=2$. Similarly, the occurrence probabilities of the two hitpatterns contributing to the 2 -hits are both equal to $1 / 2$. So, the expected value is $(2 / 4) \times(1 / 2)+(3 / 4) \times(1 / 2)=5 / 8$, which can equivalently be written as $(2 g+1) /(4 g)$ due to $g=2$.

Considering all cases of $g=3$ and any $p$ in the pulseshift, power-detection model, each of the (partial or full) hits contributed by the five hit-patterns " 001, ," "011," "100," "110," and " 111 " is counted as one full hit, and each of the (partial or full) hits contributed by the five hit-patterns " 211 ," " 221 ," "101," "121," and " 111 " is counted as two full hits. The occurrence probabilities of the five hit-patterns contributing to the 1 -hits are $5 / 24,5 / 24,3 / 16,3 / 16$, and $5 / 24$, respectively. So, the expected value becomes $(1 / 3) \times(5 / 24)+(2 / 3) \times$ $(5 / 24)+(1 / 3) \times(3 / 16)+(2 / 3) \times(3 / 16)+(3 / 3) \times(5 / 24)=$ $29 / 48 \cong 3 / 5$, which can equivalently be written as $g /(2 g-1)$ as $g=3$. Similarly, the occurrence probabilities of the five hit-patterns contributing to the 2-hit are $1 / 6,1 / 6,1 / 6$, $1 / 6$, and $1 / 3$, respectively. So, the expected value becomes $(4 / 6) \times(1 / 6)+(5 / 6) \times(1 / 6)+(2 / 6) \times(1 / 6)+(4 / 6) \times$ $(1 / 6)+(3 / 6) \times(1 / 3)=7 / 12$, which can equivalently be written as $g /(2 g-1)$ due to $g=3$.

\section{REFERENCES}

[1] G.-C. Yang and W.C. Kwong, Prime Codes With Applications to CDMA Optical and Wireless Networks, Norwood, MA: Artech House, 2002.

[2] Optical Code Division Multiple Access: Fundamentals and Applications, ed. P.R. Prucnal, Boca Raton, FL: Taylor \& Francis, 2006.

[3] W.C. Kwong and G.-C. Yang, Optical Coding Theory with Prime, New York: CRC Press, 2013.

[4] J.A. Salehi, "Code division multiple-access techniques in optical fiber networks. I. Fundamental principles," IEEE Trans. Commun., vol. 37, no. 8, pp. 824-833, Aug. 1989.

[5] W.C. Kwong, P.A. Perrier, and P.R. Prucnal, "Performance comparison of asynchronous and synchronous code-division multiple-access techniques for fiber-optic local area networks," IEEE Trans. Commun., vol. 39, no. 11, pp. 1625-1634, Nov. 1991.

[6] C.-Y. Chang, G.-C. Yang, and W.C. Kwong, "Wavelength-time codes with maximum cross-correlation function of two for multicode-keying optical CDMA," J. Lightw. Technol., vol. 24, no. 3, pp. 1093-1100, Mar. 2006.

[7] C.-Y. Chang, G.-C. Yang, C.-Y. Chang, and W.C. Kwong, "Study of a diversity O-CDMA scheme for optical wireless," J. Lightw. Technol., vol. 30, no. 10, pp. 1549-1558, May 2012.

[8] J.-W. Su, G.-C. Yang, C.-Y. Chang, and W.C. Kwong, "Design and analysis of generalized diversity optical CDMA systems," J. Lightw. Technol., vol. 31, no. 8, pp. 1217-1226, Apr. 2013.

[9] Y.-T. Lin, G.-C. Yang, C.-Y Chang, and W.C. Kwong, "Design and analysis of asynchronous incoherent optical-CDMA systems using a new code-shifting technique," IEEE Trans. Commun., vol. 63, no. 7 , pp. 2619-2631, Jul. 2015.

[10] V.J. Hernandez, A.J. Mendez, C.V. Bennett, R.M. Gagliardi, and W.J. Lennon, "Bit-error-rate analysis of a 16-user gigabit ethernet opticalCDMA (O-CDMA) technology demonstrator using wavelength/time codes," IEEE Photon. Technol. Lett., vol. 17, no. 12, pp. 2784-2786, Dec. 2005.

[11] V.J. Hernandez et al., "A 320-Gb/s capacity (32-user $\times 10 \mathrm{~Gb} / \mathrm{s}$ ) SPECTS O-CDMA network testbed with enhanced spectral efficiency through forward error correction," J. Lightw. Technol., vol. 25, no. 1, pp. 79-86, Jan. 2007.

[12] C.-S. Brès, I. Glesk, and P.R. Prucnal, "Demonstration of an eightuser 115-Gchip/s incoherent OCDMA system using supercontinuum generation and optical time gating," IEEE Photon. Technol. Lett., vol. 18, no. 7, pp. 889-891, Apr. 2006.

[13] F.R.K. Chung, J.A. Salehi, and V.K. Wei, "Optical orthogonal codes: Design, analysis, and applications," IEEE Trans. Info. Theory, vol. 35, no. 3, pp. 595-604, May 1989.

[14] W.C. Kwong, G.-C. Yang, V. Baby, C.-S Brès, and P.R. Prucnal, "Multiple-wavelength optical orthogonal codes under prime-sequence permutations for optical CDMA," IEEE Trans. Commun., vol. 53, no.1, pp. 117-123, Jan. 2005.

[15] G.-C. Yang and T. Fuja, "Optical orthogonal codes with unequal autoand cross-correlation constraints," IEEE Trans. Info. Theory, vol. 41, no. 1, pp. 96-106, Jan. 1995.

[16] T.B. Osadola, S.K. Idris, I. Glesk, and W.C Kwong, "Effect of variations in environmental temperature on 2D-WH/TS OCDMA code performance," J. Opt. Commun. Netw., vol. 5, no. 1, pp. 68-73, Jan. 2013.

[17] K. Li, W. Cong, V.J. Hernandez, R.P. Scott, J. Cao, Y. Du, J.P. Heritage, B.H. Kolner, and S.J.B. Yoo, "10 Gbit/s optical CDMA encoderdecoder BER performance using HNLF thresholder," in Proc., OSA Optical Fiber Commun. Conf. Los Angeles, CA, 23-27 Feb. 2004.

[18] W. Cong, R.P. Scott, V.J. Hernandez, K. Li, J.P. Heritage, B.H. Kolner, and S.J.B. Yoo, "High performance $70 \mathrm{Gbit} / \mathrm{s}$ SPECTS optical-CDMA network testbed," Electron. Lett., vol. 40, no. 22, pp. 1439-1440, 28 Oct. 2004.

[19] K. Kravtsov, P.R. Prucnal, and M.M. Bubnov, "Simple nonlinear interferometer-based all-optical thresholder and its applications for optical CDMA," Optics Express, vol. 15, no. 20, pp. 13114-13122, 26 Sep. 2007. 
[20] I. Glesk, P.R. Prucnal, and I. Andonovic, "Incoherent ultrafast OCDMA receiver design with 2 ps all-optical time gate to suppress multipleaccess interference," IEEE J. Select. Top. Quant. Electron., vol. 14, no. 3, pp. 861-867, May/Jun. 2008.

[21] M.D. Matinfar and J.A. Salehi, "Mathematical modeling and statistical analysis of second harmonic generation effects with thin and thick crystals in ultrahigh-speed optically amplified digital lightwave communication systems," J. Lightw. Technol., vol. 27, no. 16, pp. 3438 3452, Aug. 2009.

[22] M.R. Zefreh and J.A. Salehi, "Statistical modeling and performance characterization of ultrashort light pulse communication system using power-cubic optical nonlinear preprocessor," IEEE Trans. Commun., vol. 63, no. 8, pp. 2948-2958, Aug. 2015.

[23] M.R. Zefreh and J.A. Salehi, "Theoretical studies of ultrashort light pulse spectrally-phase-encoded OCDMA system using power-cubic optical nonlinear preprocessor," J. Lightw. Technol., vol. 33, no. 24, pp. 5062-5072, Dec. 2015.

[24] S. Galli, R. Menendez, E. Narimanov, and P. R. Prucnal, "A novel method for increasing the spectral efficiency of optical CDMA," IEEE Trans. Commun., vol. 56, no. 12, pp. 2133-2144, Dec. 2008.

[25] G.T. Kanellos, N. Pleros, C. Bintjas, H. Avramopoulos, and G. Guekos, "SOA-based interferometric optical hard-limiter," in Technical Digest, OSA Optical Amplifiers and Their Applications/Integrated Photon. Research, San Francisco, CA, 30 Jun. 2004.

[26] T.-G. Kang, D.-M. Choi, W.-K. Choi, Y.-W. Choi, S.-J. Kim, and W.K. Choi, "Experimental investigation of optical thyristor operating as optical hard-limiter," Microwave Optical Techn. Lett., vol. 49, no. 11, pp. 2779-2780, Nov. 2007.

[27] T. Ohtsuki, K. Sato, I. Sasase, and S. Mori, "Direct-detection optical synchronous CDMA systems with double optical hard-limiters using modified prime sequence codes," IEEE Trans. Commun., vol. 14, no. 9, pp. 1879-1887, Dec. 1996

[28] J.-J. Chen and G.-C. Yang, "CDMA fiber-optic systems with optical hard limiters," IEEE J. Lightw. Technol., vol. 18, no. 7, pp. 950-958, Jul. 2001.

[29] C.-C. Hsu, G.-C. Yang, and W. C. Kwong, "Hard-limiting performance analysis of 2-D optical codes under the chip-asynchronous assumption," IEEE Trans. Commun., vol. 56, no. 5, pp. 762-768, May 2008.

[30] N.T. Dang, A.T. Pham, and Z. Cheng, "Beat noise cancellation in 2-D optical code-division multiple-access systems using optical hard-limiter array," IEICE Trans. Commun., vol. E93-B, no. 2, pp. 289-292, Feb. 2010

[31] I. Glesk, M. Sorel, A.E. Kelly, and P.R. Prucnal, "Enhancing performance of optical communication systems with advanced optical signal processing," Special Issue on All-Optically Routed Networks, J. Networks, vol. 5, no. 11, pp. 1328-1333, Nov. 2010.

Chin-Yi Tsai received the B.S. degree in electrical engineering from the ???? University, ????, Taiwan, in 201?, and the M.S. degree in electrical engineering from the National Chung Hsing University, Taichung, Taiwan, in 2017.

His research interests include optical communications.

Guu-Chang Yang (S'88-M'92-SM'05-F'12) received the B.S. degree from National Taiwan University, Taipei, Taiwan, in 1985, and the M.S. and Ph.D. degrees from the University of Maryland, College Park, MD, in 1989 and 1992, respectively, all in electrical engineering.
From 1988 to 1992, he was a Research Assistant in the System Research Center, University of Maryland. In 1992, he joined the faculty of the Nationa Chung Hsing University, Taichung, Taiwan, where he is currently a Chair Professor with the Department of Electrical Engineering and the Graduate Institute of Communication Engineering. He was the Chairman of the Department of Electrical Engineering from 2001 to 2004. He has co-authored the first-of-its-kind technical book on optical coding theory and its applications to code-division multiple access (CDMA), Prime Codes with Applications to CDMA Optical and Wireless Networks (Norwood, MA, USA: Artech House, 2002). He has contributed one chapter on optical codes to another technical book, Optical Code Division Multiple Access: Fundamentals and Applications (Boca Raton, FL, USA: Taylor \& Francis, 2006). He has co-authored the classical reference book, Optical Coding Theory with Prime (New York, NY, USA: CRC Press, 2013). His research interests include wireless and optical communication systems, modulation and signal processing techniques, and applications of CDMA.

Dr. Yang is currently an Associate Editor of the IEEE TRAnsaCtions ON COMMUniCATIONS, and serves as the Co-Coordinator of the Ministry of Science and Technology's Development and Applications of Advanced Communications Networking Technologies Program from 2014 to 2017. He served as the Area Coordinator of the Ministry of Science and Technology's Telecommunications Program in 2014, the Area Coordinator of the National Science Council's Telecommunications Program from 2012 to 2013, and the Co-Coordinator of the National Science Council's National Networked Communication Program from 2010 to 2014. He also served as the Chairman of the IEEE Communications Society (Taipei Chapter) from 2013 to 2014 the Vice Chairman of the IEEE Communications Society (Taipei Chapter) from 2011 to 2012, the Chairman of the IEEE Information Theory Society (Taipei Chapter) from 2003 to 2005, and the Vice Chairman of the IEEE Information Theory Society (Taipei Chapter) from 1999 to 2000. He became an IEEE Fellow in 2012 for contributions to optical CDMA. He received the Distinguished Research Award from the National Science Council in 2004 and 2014, and the Outstanding Young Electrical Engineer Award in 2003 and the Distinguished Electrical Engineering Professor Award in 2012, both from the Chinese Institute of Electrical Engineering. He also received the Best Teaching Award from the Department of Electrical Engineering, National Chung Hsing University from 2001 to 2004 and in 2008.

Jing-Shiuan Lin received the B.S. degree in electrical engineering from the Tamkang University, New Taipei City, Taiwan, in 2013, and the M.S. degree in electrical engineering from the National Chung Hsing University, Taichung, Taiwan, in 2015.

His research interests include optical communications.
Cheng-Yuan Chang (S'04-M'07) received the B.S. degree from National Sun Yat-Sen University, Kaohsiung, Taiwan, in 1997, and the M.S. and Ph.D. degrees from National Chung Hsing University, Taichung, Taiwan, in 2002 and 2007, respectively, all in electrical engineering.

In 2007, he joined the faculty of National United University, Miaoli, Taiwan, where he is presently an Associate Professor with the Department of Electrical Engineering. His research interests include optical and wireless communications, spreading code designs, and modulation and coding techniques.

Ivan Glesk (M?01?SM?01) received the Ph.D. degree in quantum electronics and optics from Comenius University, Bratislava, Slovak Republic, in 1989, and the D.Sc. degree from the Slovak Academy of Sciences, Bratislava.

In 1986, he joined the Department of Experimental Physics, Comenius University, where he later became a professor of physics, and was engaged in research in the areas of nonlinear optics, laser physics, and light detection and ranging (LIDAR) methods of remotely sensing the atmosphere. In 1991, he joined Princeton University, USA, where he was Manager of the Lightwave Communication Research Laboratory and Senior Research Scholar in the Department of Electrical Engineering. In 2007, he joined University of Strathclyde, Glasgow, UK, as Professor of Broadband Communication Systems in the Department of Electronic and Electrical Engineering. His current research interests include applications of ultra-short pulsed laser systems for optical networks, ultrafast all-optical signal processing, and optical interconnects. 
$\mathrm{He}$ is author or coauthor of more than 280 publications and 16 book chapters and holds five patents. He is on the editorial board of the International Journal of Optics and Optica Applicata and is a recipient of the International Research and Exchanges Board (IREX) Fellowship.

Wing C. Kwong (S'88-M'92-SM'97) received the B.S. degree from the University of California, San Diego, CA, in 1987, and the Ph.D. degree from Princeton University, Princeton, NJ, in 1992, both in electrical engineering.

ince 1992, he has been a faculty member at Hofstra University, Hempstead, NY, where he is currently a Professor with the Department of Engineering and the School of Engineering and Applied Science. He has co-authored the first-of-its-kind technical book on optical coding theory and its applications to code-division multiple access (CDMA), Prime Codes with Applications to CDMA Optical and Wireless Networks (Norwood, MA, USA: Artech House, 2002). He has contributed one chapter on optical codes to another technical book, Optical Code Division Multiple Access: Fundamentals and Applications (Boca Raton, FL, USA: Taylor \& Francis, 2006). He has co-authored the classical reference book, Optical Coding Theory with Prime (New York, NY, USA: CRC Press, 2013). He has authored numerous professional articles. He chaired technical sessions and served technical program committees in international conferences. He has given seminars and tutorials in optical coding theory and its applications in various cities and countries, such as Montreal (Canada), Hong Kong (China), Seoul (South Korea), Hsinchu and Taichung (Taiwan), and Princeton (NJ, USA). His research interests include code theory, optical and wireless communication systems and multiple-access networks, and ultrafast all-optical signal processing techniques.
Dr. Kwong was the recipient of the NEC Graduate Fellowship awarded by NEC Research Institute, Princeton, NJ, USA in 1990 and 1991. He received the Young Engineer Award from the IEEE (Long Island chapter) in 1998. He received the 2016 Teacher of the Year award from the School of Engineering and Applied Sciences, Hofstra University. He was an Associate Editor of the IEEE TRANSACTIONS ON COMMUNICATIONS. 